\title{
A mechanistic description of the formation and evolution of vegetation patterns
}

\author{
R. Foti ${ }^{1, *}$ and J. A. Ramírez ${ }^{1}$ \\ ${ }^{1}$ Department of Civil and Environmental Engineering, Colorado State University, Fort Collins, Colorado, USA \\ *now at: Department of Civil and Environmental Engineering, Princeton University, Princeton, New Jersey, USA
}

Correspondence to: R. Foti (rfoti@princeton.edu)

Received: 6 June 2012 - Published in Hydrol. Earth Syst. Sci. Discuss.: 19 July 2012

Revised: 2 December 2012 - Accepted: 11 December 2012 - Published: 11 January 2013

\begin{abstract}
Vegetation patterns are a common and welldefined characteristic of many landscapes. In this paper we explore some of the physical mechanisms responsible for the establishment of self-organized, non-random vegetation patterns that arise at the hillslope scale in many areas of the world, especially in arid and semi-arid regions. In doing so, we provide a fundamental mechanistic understanding of the dynamics of vegetation pattern formation and development. Reciprocal effects of vegetation on the hillslope thermodynamics, runoff production and run-on infiltration, root density, surface albedo and soil moisture content are analyzed. In particular, we: (1) present a physically based mechanistic description of processes leading to vegetation pattern formation; (2) quantify the relative impact of each process on pattern formation; and (3) describe the relationships between vegetation patterns and the climatic, hydraulic and topographic characteristics of the system. We validate the model by comparing simulations with observed natural patterns in the areas of Niger near Niamey and Somalia near Garoowe. Our analyses suggest that the phenomenon of pattern formation is primarily driven by run-on infiltration and mechanisms of facilitation/inhibition among adjacent vegetation groups, mediated by vegetation effects on soil properties and controls on soil moisture and albedo. Nonetheless, even in presence of those mechanisms, patterns arise only when the climatic conditions, particularly annual precipitation and net radiation, are favorable.
\end{abstract}

\section{Introduction}

The presence of self-organized vegetation patterns is a common and well-defined characteristic of many arid and semiarid landscapes. Indeed, vegetation is in general spatially heterogeneous and its constituent species show spatial distributions that depart from complete randomness (Greig-Smith, 1979), although only in a few cases, where this departure is more marked, the pattern structure is easily recognizable.

Vegetation patterns exhibit a multitude of shapes (banded, spotted or labyrinthine) and occur at a wide variety of spatial scales. Typical dimensions of a vegetation pattern element (i.e., width of a band or radius of a patch of vegetation) can span up to two orders of magnitude, ranging from $10^{\circ} \mathrm{m}$ to almost $10^{2} \mathrm{~m}$ (Rietkerk and Van de Koppel, 2008).

The identification and characterization of this phenomenon, as well as the individuation of the processes responsible for specific types of these patterns (e.g., so-called tiger bushes) were the main focus of numerous studies (e.g., (Greig-Smith, 1979; Dunkerley and Brown, 1999; Thiéry et al., 1995; Worral, 1959). Initially, studies were mainly focused on qualitative descriptions and on identifying and listing the recurrence of certain types of spatial configurations (Boaler and Hodge, 1962; Worral, 1960). However, more recently, research has been directed to a more quantitative characterization of the processes leading to the formation of vegetation patterns (D’Odorico et al., 2006; Lefever and Lejeune, 1997). Most of these studies agree that the development of non-random self-organized configurations is the result of short-range synergy and long-range competition occurring between plants and groups of plants (D'Odorico et al., 2006; Valentin et al., 1999). 
Plants, especially in arid landscapes, help reduce soil erosion and augment soil permeability; they also protect each other from winds and damage caused by animals and extreme temperatures and humidity conditions. In some areas, these interactions favor the formation of bands of vegetation perpendicular to the planar slope vector in mild hillslopes (Bromley et al., 1997) or perpendicular to the direction of the prevalent winds in response to their erosive action (Leprun, 1999). Although hillslope-scale patterns can arise in a variety of regions and climates, scarcity of water seems to be the common denominator of every landscape characterized by observable vegetation patterns.

Many models have been developed to describe vegetation structures that deviate from randomness. Cellular automata models, for example, have been successfully adopted to investigate the spatial distribution of Acacia trees in desertic areas, (Wiegand et al., 1999, 2000) or to analyze gap dynamics and cohesistence of trees and grass in savannas (Jeltsch et al., 1996). However, most of the models that have been used specifically to reproduce the geometric vegetation structures that are the objects of this study belong to three categories: (1) kernel based models (Lefever and Lejeune, 1997; Thiéry et al., 1995; D'Odorico et al., 2006); (2) advectiondiffusion models (HilleRisLambers et al., 2001; Rietkerk et al., 2002); and (3) differential flow instability models (Klausmeier, 1999; Sherrat, 2005; Saco et al., 2007). With this effort, we intend to investigate vegetation pattern formation using a mechanistic water balance model coupled to phenomenological conceptualizations of the feedbacks among the components of the climate-soil-vegetation system. Our overarching objective is to explore and identify (some of) the physical mechanisms responsible for the establishment of non-random spatial vegetation patterns that arise in arid and semi-arid regions. In order to do so, we (1) develop a physically based mechanistic understanding of the processes leading to vegetation pattern formation; (2) implement such understanding in a mathematical model able to replicate the main physical characteristics of observed vegetation patterns; (3) individuate the relative importance of each process in pattern formation; and (4) capture the relationships between vegetation patterns and the climatic, hydraulic and topographic characteristics of the system.

\section{Hypotheses}

By "vegetation patterns" we refer to the non-random arrangement of vegetated and bare patches of soil on the landscape, where non-randomness indicates any spatial distribution of patches that deviates from a purely spatially random distribution.

Our first hypothesis is that vegetation patterns emerge as a result of physical, chemical and physiological feedbacks between vegetation, hydrologic and climatic processes, and soil
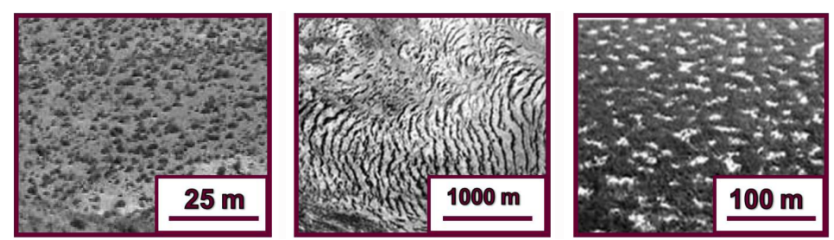

Fig. 1. Vegetation patterns typical shapes and dimensions (edited from D'Odorico et al., 2006).

properties, and that those feedback processes are amenable to quantitative description and modeling.

Our second hypothesis is that patterns develop because those feedback processes tend to make certain regions in the neighborhood of an existing clump of vegetation more conducive to the establishment of additional vegetation (or not).

Finally, our third hypothesis is that the spatial distribution of vegetation depends on the spatial distribution of soil moisture and energy (which, in turn, is influenced by the vegetation itself). Thus, physiological and hydrological processes conducive to local decreases in the available soil water and nutrients will tend to inhibit vegetation establishment and those conducive to locally maintaining or increasing soil water and nutrients will tend to promote vegetation establishment.

In general, the previous hypotheses imply that the spatial structuring of vegetation is the result of a series of process that optimize the use of the available resources (Schymanski et al., 2009, 2010)

\section{Methods}

We are interested in analyzing vegetation agglomerates emerging at the hillslope scale and whose typical dimensions are of the order of magnitude of $10^{0}$ to almost $10^{2} \mathrm{~m}$ (Fig. 1). Hence, we simulate the climate-soil-vegetation dynamics of a hillslope on a two-dimensional gridded domain of area $10^{5}-10^{7} \mathrm{~m}^{2}$. In order to characterize self-organized spatial structures, we subdivide the study domain into a grid of pixels of area $10^{0}-10^{2} \mathrm{~m}^{2}$.

Water and energy fluxes occur mainly in the vertical direction, across the interface between soil and atmosphere. Spatial interactions between vegetation agglomerates, and mutual interactions between soil, water fluxes, and vegetation, on the other hand, mostly occur in the horizontal directions. Therefore, the description of these coupled processes requires a 3-D dynamical model. However, following a successful approach used to investigate the impact of climate on the vegetation in drylands (Tietjen et al., 2009), here we model these 3-D interactions by means of a combination of a 1-D water balance model to describe the vertical fluxes, coupled to a 2-D model to capture the horizontal interactions. 
Although observed vegetation patterns show variability over time, their statistical properties do not change drastically within a year and from year to year, suggesting that patterns are the result of adaptation to the long-term average characteristics of the soil-climate system rather than a response to short-term disturbances. Under the assumption of stationary climate, therefore, we use long-term average climatic and hydraulic conditions in order to determine the spatial configurations of vegetation and associated water and energy fluxes that are in long-term equilibrium with the soil-climate system.

\subsection{Procedure schematization}

Because the long-term average vegetation density (i.e., the portion of a given area which is covered by vegetation) at a certain location in space is the long-term response of the climate-soil-vegetation system to a set of environmental forcings (e.g., precipitation, temperature, solar radiation), knowledge of the spatial arrangement of the environmental forcings over a certain domain can be used to determine the configuration of vegetation density over the same domain. Therefore, we seek to determine a spatial configuration of fluxes and vegetation density that simultaneously satisfies the water and energy budgets both at the global (i.e., for the entire study domain) and the local (i.e., for each pixel) scales, while taking into account lateral interactions (i.e., between adjacent pixels) between vegetation, climate and soil.

However, the aforementioned spatial configuration of vegetation and water fluxes across the study hillslope is, a priori, unknown. In order to find it, that is, in order to determine the local-scale fluxes and vegetation density that are in equilibrium with the hillslope-scale conditions, we use an iterative procedure. For a given set of initial climatic conditions and soil properties, long-term averages of annual fluxes of water and latent heat of evapotranspiration, as well as of vegetation density, are estimated at the local scale for each one of the pixels of the study domain. Once the vertical fluxes and vegetal density are estimated at the local scale through the vertical water budget, the mutual lateral effects of vegetation and fluxes are evaluated. As mentioned before, those mutual effects are predicated on the assumption that vegetation and fluxes exert mutual feedback within the hillslope. Evaluation of lateral effects allows estimation of an updated set of inputs (e.g., water input from uphill, feedback of soil moisture on albedo) and soil parameters (e.g., feedback of vegetation density on soil hydraulic conductivity) that are used to perform the subsequent iteration. A flow chart of the simulation procedure is provided in Fig. 2.

Because the configuration of the local (i.e., at the pixel scale) water fluxes is obtained by redistributing the available global (i.e., of the entire domain) water input, the global water budget is actually satisfied at each step of the iterative procedure. Analogous reasoning can be made with respect to the latent heat evapotranspiration flux.

\subsection{1-D water budget: vertical fluxes and forcings}

The water budget at any pixel of our study domain is quantified using a one-dimensional physically based mechanistic representation of soil moisture dynamics as forced by a stochastic climate (Eagleson, 1978a, b, c, d, e, f, g). It describes the relationship between annual amounts of precipitation, runoff (both surface and groundwater), infiltration and evapotranspiration as a function of volumetric soil moisture and soil and vegetation characteristics (additional details are provided in Appendix). In doing so, the model assumes that the soil-vegetation system is in a long-term equilibrium with climate, and that the value of long-term equilibrium soil moisture maximizes vegetal biomass and minimizes vegetation stress (Eagleson, 1978f). It is indeed the assumption of long-term climate-soil-vegetation equilibrium that made Eagleson's model our optimal choice for the characterization of the 1-D vertical dynamics. Although it can be argued that the short-term behavior of the biotic system is driven by short-term climatic forcings (e.g., few large pulses of precipitation), the vegetation patterns we are interested in are observed across long time spans, suggesting that they are the result of a long-term adaptation process.

Eagleson's water balance model characterizes the soil in terms of the following hydraulic parameters: total porosity, pore size distribution index, surface retention capacity, saturated hydraulic conductivity and saturated matric potential. The model describes the climate drivers as a function of: mean storm intensity, mean storm duration, mean time between storms, rainy season length, mean and variance of storm depth, mean annual precipitation and mean annual potential evapotranspiration.

In Eagleson's model, the role of vegetation in the water balance is captured through the plant transpiration efficiency, $k_{v}$, defined as the ratio between the potential rate of transpiration to the potential rate of bare soil evaporation and through the fractional vegetation cover or vegetation density, $M$.

The output of the model is characterized by long-term averages of the water fluxes (i.e., evapotranspiration, surface runoff and groundwater runoff) as well as the long-term equilibrium soil moisture content and the vegetation density. The estimation of vegetation density and water fluxes is predicated on the assumption that the soil-vegetation system is in a long-term equilibrium with climate, and that the value of long-term equilibrium soil moisture maximizes vegetal biomass and minimizes vegetation stress (Eagleson, 1978f). The water balance model is summarized in Eqs. (1)-(22) of Eagleson (1978f).

\subsection{2-D spatial feedbacks characterization: horizontal fluxes and interactions}

The climate-soil-vegetation system is very complex and governed by strong feedbacks between all elements of the system. Plants affect the physical structure of the 


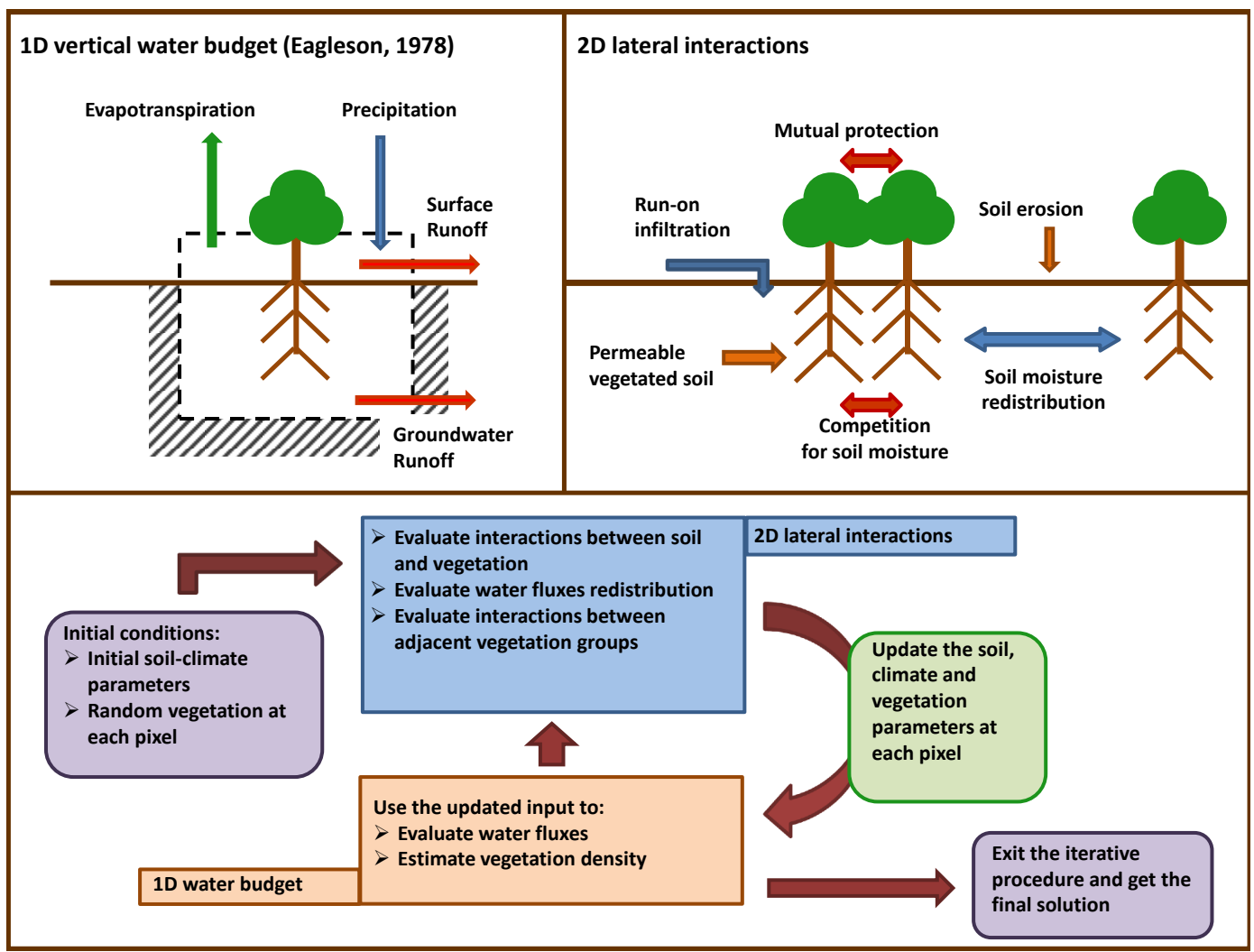

Fig. 2. Schematic of the 1-D vertical budget model and the 2-D lateral interactions with flowchart of the simulation procedure of the soilclimate-vegetation system.

soil-vegetation system by perturbing the thermal and aerodynamic properties of the canopy layer as well as the soil structure (i.e., texture, porosity, connectivity, hydraulic conductivity, etc.). These perturbations in turn lead to changes in the water and energy fluxes in the neighborhood of the plant that may promote or inhibit the establishment of surrounding vegetation. We focus only on a subset of factors that we hypothesize are the main drivers of the process of vegetation pattern formation and evolution. These factors are: (1) modification of the spatial distribution of soil hydraulic conductivity by vegetation, (2) infiltration of surface runoff, a phenomenon known as run-on infiltration, (3) spatial reconfiguration of soil albedo, (4) spatial soil moisture redistribution due to roots, and (5) redistribution of nutrients.

Although fire, livestock, and other such external forces may be the main cause determining vegetation patterns in some instances, vegetation patterns as those shown in Fig. 1 are observed even in the absence of such forces. Therefore, our work focuses on the feedbacks and interactions between vegetation, soil, and hydro-climatic processes only.

\subsubsection{Effect of vegetation on soil hydraulic conductivity}

The soil hydraulic characteristics vary depending on the presence or absence of vegetation and on the evolution of vege- tation density. Plants influence erosion and sediment transport by limiting the effect of wind and slowing the surface runoff velocity, therefore constituting areas of potential sediment accumulation. In addition, the superficial soil of a vegetated area is much richer in litter and organic debris, therefore it is richer in nutrients and more porous and permeable. Permeability of deeper layers is also affected by the presence of roots and rotting roots, which create preferential routes for infiltrated water (Boaler and Hodge, 1962; Bromley et al., 1997). All of these effects have been observed in areas characterized by vegetation patterns, where vegetated soil exhibits higher permeability than adjacent bare soil, which often has a highly impermeable superficial crust (Valentin et al., 1999). The range of hydraulic conductivity of an area characterized by vegetation patterns can be very wide, often spanning several orders of magnitude and subjected to random variations within very short distances (Bromley et al., 1997). Soil permeability at a site, therefore, is a function of vegetation density (see schematization in Fig. 3a).

We model the saturated hydraulic conductivity as a continuous function of vegetation density $M_{X}$ at the given point $X$ as follows:

$K_{\mathrm{S} X}=\sum_{i=1}^{i=10}\left[a_{i}+b_{i} \cdot\left(M_{X}-0.1 \cdot i\right) \cdot I_{[0.1(i-1), 0.1 i)}\left(M_{X}\right)\right]$ 


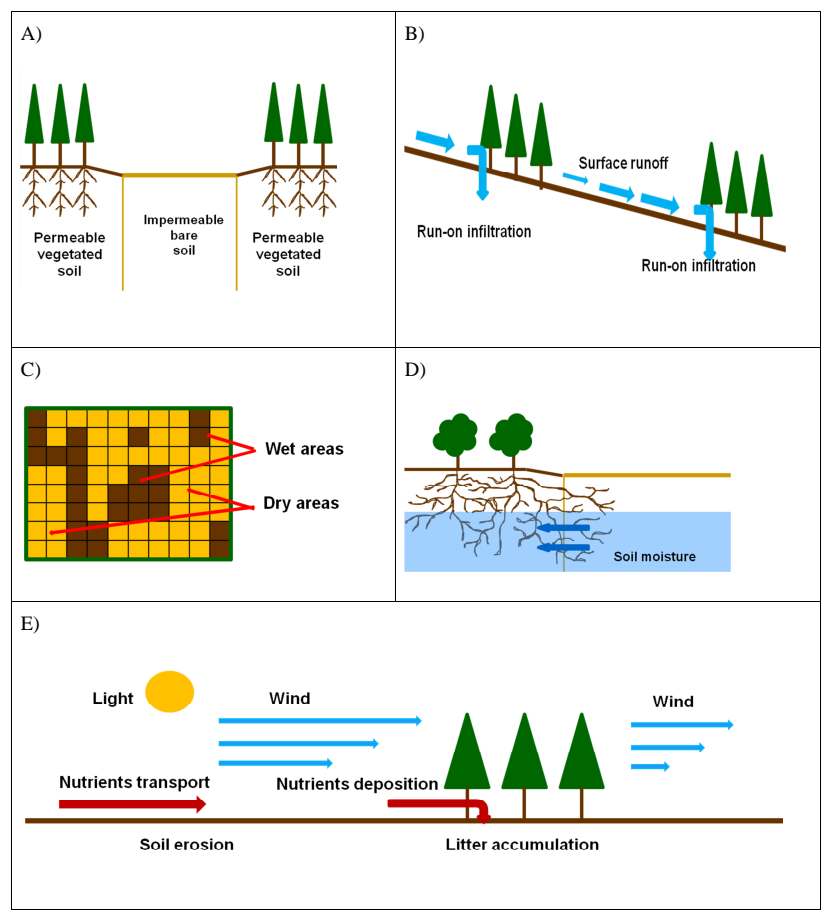

Fig. 3. Qualitative schematization of the interactions between soil, climate and vegetation. (A) Effect of vegetation on soil permeability. (B) Effect of vegetation on run-on infiltration. (C) Effect of soil moisture on soil reflectance. (D) Effect of roots on soil moisture. (E) Other spatial interactions (effect of vegetation on wind, light, nutrients, etc.).

where $I_{[\alpha, \beta)}(M)$ is the indicator function such that:

$I_{[\alpha, \beta)}(M)=\left\{\begin{array}{l}1, \alpha \leq M<\beta \\ 0, \text { otherwise } \\ .\end{array}\right.$

The choice of the coefficients $a_{i}$ and $b_{i}$ of Eq. (1) is aimed at obtaining a piecewise continuous function spanning a range of saturated hydraulic conductivity values compatible with observations as reported in the literature (Bromley et al., 1997) which, for the sites where vegetation patterns emerge, are typically found in the interval $10^{-6}$ to $10^{-3} \mathrm{~cm} \mathrm{~s}^{-1}$ over a range of vegetation density ranging between 0 and 1 .

\subsubsection{Run-on infiltration of surface runoff}

A non-uniform spatial distribution of hydraulic conductivity affects both the vertical water fluxes as well as the water input of downstream points through the process of run-on infiltration. Surface runoff plays a key factor in the development of soil and vegetation. Part of the surface runoff can pond in small depressions or be trapped in areas of litter deposition downhill and infiltrate (Bromley et al., 1997) (see also schematization in Fig. 3b). The amount of surface runoff that
A)

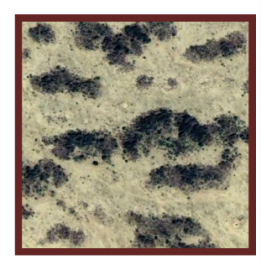

D)

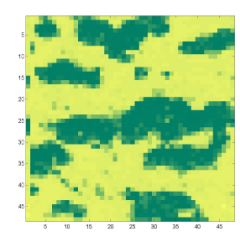

G)

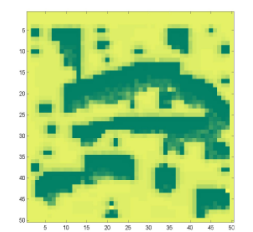

B)

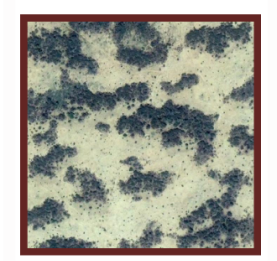

E)

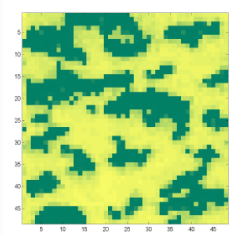

H)

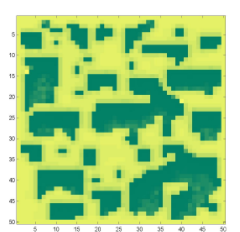

C)

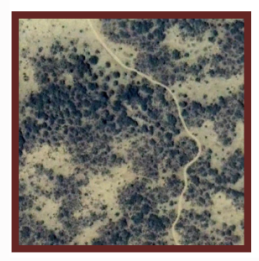

F)

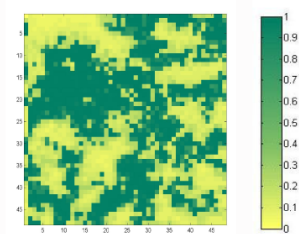

I)

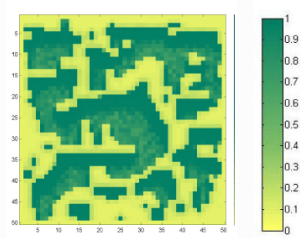

Fig. 4. (A), (B), (C) Aerial photographs of natural patterns (Tiger Bushes) in Niger $\left(13^{\circ} 20^{\prime} \mathrm{N}, 2^{\circ} 04^{\prime} \mathrm{E}\right)$. (D), (E), (F) Digitized representation of the vegetation covers of the natural patterns corresponding respectively to (A), (B) and (C). (G), (H), (I) Simulated patterns.

infiltrates depends on many factors, such as soil properties, topography, overall water input, characteristics of the rain event and so on. The runoff produced accumulates along the hillslope, causing erosion and sediment transport.

Thus, the long-term water balance at each point $X$ considers a water input, $P_{X}$, given by the sum of the long-term average precipitation $m_{\mathrm{PA}}$ at $X$ and the average surface runoff, $R_{\mathrm{S} Y}$, coming from the uphill location $Y$ :

$P_{X}=m_{\mathrm{PA}}+R_{\mathrm{S}} Y$,

$Y=X+\nabla F_{X} \cdot \mathrm{d} s$

where $F_{X}$ is the topographic elevation function of the domain evaluated at the point $X$ and $\nabla$ is the gradient operator.

\subsubsection{Effect of vegetation on albedo}

Albedo is a characteristic of the reflecting surface and, among other things, depends on soil moisture and on vegetation characteristics including vegetation density. Wetter and more densely vegetated soils are usually darker and less reflective (Fig. 3c) and, therefore, are characterized by lower albedo. While albedo may have both a negative and a positive dependence on vegetation density depending on the color of bare soil that characterizes the region of study (Rechid et al., 2009), its dependence on soil moisture is clearer, wet terrain usually being less reflective than dry terrain (Lobell 
and Asner, 2002; Wang et al., 2005). For soil-vegetation systems, less absorbed net energy means less available energy for sensible heating and for evaporating water. Consequently, all else being equal, higher albedo corresponds to lower potential rate of evapotranspiration.

We express the total albedo, $\rho_{\mathrm{T}}$, as an exponentially decreasing function of the long-term mean of soil moisture such that:

$\rho_{\mathrm{T} X}=\rho_{\mathrm{T} X}^{*}-A \cdot\left[e^{-s_{0 X} / B}-e^{-1 / B}\right]$,

where $\rho_{\mathrm{T}}^{*}$ is the total (i.e., all-frequency) surface albedo for saturated soil and $s_{0 X}$ is the soil moisture, while $A$ and $B$ are coefficients whose value depends on the properties of the surface. This formulation accounts explicitly for the dependence of albedo on soil moisture and implicitly for its dependence on vegetation through the dependence of soil moisture on vegetation density. For our simulations, $\rho_{\mathrm{T}}^{*}$ was set to 0.25 , $A$ to 0.11 and $B$ to 0.3 (Lobell and Asner, 2002).

\subsubsection{Soil moisture redistribution by roots}

The root configuration is peculiar of each vegetation species and is affected by the plant's age and health, as well as the soil characteristics, water availability, temperature, and other environmental factors. Developing a model of moisture redistribution by roots that encodes all of these spatially and temporally varying effects is a complex task that goes beyond the scope of this work. We propose a basic approach based on the simplifying assumption that the root characteristics of the vegetation populating the domain are spatially uniform and that soil moisture can be rerouted out of a pixel into another by root networks only if they extend across the pixel borders (Fig. 3d). We model the net exchange of water input, $P_{X}$, at each point as:

$$
\left.\frac{\mathrm{d} P}{\mathrm{~d} \boldsymbol{X}}\right|_{X}=\left.\frac{\xi_{R}-1}{4} \cdot \frac{\mathrm{d}\left(M \cdot k_{v} \cdot P\right)}{\mathrm{d} X}\right|_{X},
$$

where $k_{v}$ represents the transpiration efficiency and $\xi_{R}$ represents the degree to which roots extend over the four adjacent pixels as the ratio of the rooted area to the characteristic area of the pixel:

$\xi_{R}=\frac{A_{\text {roots }}}{A_{\text {cell }}}$

The parameter $\xi_{R}$ defines a range of root actions and allows us to take into account the process of subsurface water transfer between adjacent pixels promoted by root systems.

Equation (5) implies that roots spreading across the borders of a cell can uptake a fraction of the soil moisture of the neighboring cell in a way that is proportional (proportionality being given by the parameter $\xi_{R}$ ) to the vegetation density of the contiguous pixels and to their transpiration efficiencies.

\subsubsection{Effect of vegetation on transpiration efficiency}

The interactions between individual plants are multifold and may lead to positive and negative feedbacks on vegetation density. While, on the one hand, plants compete for water and nutrients through roots and for light through foliage (Barbier et al., 2008; Holmgren et al., 1997), they can also protect each other from extreme fluctuations of temperature and humidity, from the action of surface runoff, from mechanical or herbivore damages, and can improve soil properties through litter formation, augmented soil porosity and nutrient replenishment (Holmgren et al., 1997; Borgogno et al., 2009) (see also Fig. 3e).

In this study we express the cumulative effect of those interactions as a function of their impact on the transpiration efficiency, $k_{v}$, following the reasoning that the net result of facilitation/competition should be to improve/worsen water use efficiency by increasing/decreasing the quantity of biomass that can be produced out of a certain amount of transpired water.

Therefore, we model the transpiration efficiency, $k_{v X}$, at a certain point $X$, as a function of: a base value for the transpiration efficiency, $\overline{k_{v}}$; the local vegetation density, $M_{X}$; the vegetation density of surrounding points, $M_{\mathrm{U}}, M_{\mathrm{D}}, M_{\mathrm{L}}, M_{\mathrm{R}}$, the hillslope-scale vegetation density at the initial time step, $\bar{M}$; the local surface runoff, $R_{\mathrm{S} X}$; the surface runoff of immediately upslope and downslope, $R_{\mathrm{s} U}, R_{\mathrm{s} D}$; and the hillslopescale average surface runoff for uniform vegetation density at the initial step, $\overline{R_{\mathrm{s}}}$. This function has the following form:

$$
\begin{aligned}
k_{v X} & =\max \left\{0.5, \min \left\{1, \overline{k_{v}}+\left[\left(\Delta k_{v X}\right)_{1}+\left(\Delta k_{v X}\right)_{2}\right.\right.\right. \\
& \left.\left.\left.+\left(+\left(\Delta k_{v X}\right)_{3} \Delta k_{v X}\right)_{4}+\left(\Delta k_{v X}\right)_{5}\right]\right\}\right\},
\end{aligned}
$$

where:

$$
\begin{aligned}
& \left(\Delta k_{v X}\right)_{1}=-\alpha_{1} \cdot \frac{M_{X}-\bar{M}}{\bar{M}} \\
& \left(\Delta k_{v X}\right)_{2}=-\alpha_{2} \cdot \frac{M_{U}+M_{D}+M_{L}+M_{R}-4 \cdot \bar{M}}{\bar{M}}, \\
& \text { where }\left\{\begin{array}{l}
U=X+\nabla F_{X} \cdot \mathrm{d} s \\
D=X-\nabla F_{X} \cdot \mathrm{d} s \\
L=X+\Theta_{X} \cdot \mathrm{d} s \\
R=X-\Theta_{X} \cdot \mathrm{d} s \\
\text { where } \Theta_{X} \perp \nabla F
\end{array}\right.
\end{aligned}
$$

$\left(\Delta k_{v X}\right)_{3}=-\alpha_{3} \cdot \frac{\overline{R_{\mathrm{s}}}-R_{\mathrm{s} X}}{m_{\mathrm{PA}}}$

$\left(\Delta k_{v X}\right)_{4}=-\alpha_{4} \cdot \frac{R_{\mathrm{s} U}-R_{\mathrm{S} X}}{m_{\mathrm{PA}}}$, where $U=X+\nabla F_{X} \cdot \mathrm{d} s$

$\left(\Delta k_{v X}\right)_{5}=\alpha_{5} \cdot \frac{R_{\mathrm{s} D}-R_{\mathrm{s} X}}{m_{\mathrm{PA}}}$, where $D=X-\nabla F_{X} \cdot \mathrm{d} s$ 
Equations (8) and (9) describe the change of the local transpiration efficiency at point $X$ as a function of the vegetation density at both the point itself and at the adjacent ones. The presence of the negative sign in Eqs. (8) and (9) derives from the fact that the presence of vegetation in the given pixel and in its neighborhoods is assumed to produce a facilitation effect for further establishment of vegetation.

Equations (10), (11) and (12), on the other hand, reflect the effect of surface runoff through the processes of erosion and sedimentation of both soil particles and nutrients. The negative sign in Eqs. (10) and (11) are suggested by the following considerations: (1) when a given location (i.e., pixel) is subjected to a surface runoff, $R_{\mathrm{S} X}$, larger than the average surface runoff corresponding to uniform density, $\overline{R_{\mathrm{S}}}$, it is concurrently subjected to soil erosion and nutrients deprivation; (2) when a given pixel is subjected to a surface runoff, $R_{\mathrm{S} X}$, lower than the surface runoff of the upstream pixel, $R_{\mathrm{S} U}$, it benefits from the partial deposition of incoming soil particles and nutrients and (3) when a given pixel is subjected to a surface runoff, $R_{\mathrm{S} X}$, lower than the downstream pixel, $R_{\mathrm{S} U}$, it is subjected to an accelerated superficial flow which exposes it to nutrients and soil loss.

\section{Simulation of the system}

The climate-soil-vegetation system was simulated under various combinations of climatic forcing, soil parameters and lateral interaction functions (Eqs. 8 through 12) in order to explore the conditions controlling the mechanism of pattern emergence and evolution. We present results of the simulation of the system on a study domain of 50x50 pixels, representing a hillslope of about $10^{5} \mathrm{~m}^{2}$. Boundary conditions of the system are: (1) for all domain borders, fractional vegetation coverage is kept equal to the initial uniform solution (obtained by using the domain-averaged inputs and equal to the vegetal density of each pixel at the preliminary step of simulation); (2) for upstream and lateral borders, water fluxes are kept equal to the initial uniform solution; and (3) free flow condition for the downstream boundary, allowing complete drainage downhill.

We used the model both to simulate real sites characterized by the presence of vegetation patterns and for a non site-specific system (the latter in order to evaluate the system sensitivities to each mechanism considered.) Unless otherwise stated, simulations were carried out on a constant slope domain whose hydraulic properties and climatic forcing are reported in the "base conditions" column of Table 1. In order to incorporate the typical random spatial variability of soil conductivity (Bromley et al., 1997), a random component is superimposed to the value of $K_{\mathrm{s} X}(n)$ obtained with Eq. (1).

\section{Results and discussion}

\subsection{Spatial analysis}

In order to compare different typologies of patterns and objectively measure the individual impact of the climatic and hydraulic properties of the system on pattern emergence and characteristics, we explore the following spatial characteristics of vegetation fields:

- Probability density functions (PDFs) and conditional PDFs of vegetation density at the pixel level.

- Power spectral density functions of the vegetation density fields.

- Number, size and shape of vegetation clusters.

\subsubsection{PDFs and conditional PDFs of vegetation density}

PDFs of the vegetation density at the pixel scale can be used to evaluate how different a given vegetation field is from the typical field that would be produced if the plants were distributed randomly and independently in space. In this case, we may assume that the vegetation density of each pixel is a one to one function of the number of plants present within the pixel itself. Following this assumption, the PDF of the pixel vegetation density throughout the domain would be normally distributed with mean equal to the average pixel vegetation coverage $^{1}$. For self-organized vegetation patterns, the presence of a clump of vegetation at a certain point in space has an impact on the vegetation establishment in its neighborhood. Therefore, presence of self-organized structures can be inferred from the analysis of conditional PDFs of the vegetation density. We do so by evaluating the PDF of vegetation density for all those pixels having at least one neighbor characterized by vegetation density higher than the overall domain average. The same conditioning is done on the neighborhood of a pixel characterized by vegetation density lower than the domain average. In order to detect spatial anisotropy, conditional PDFs are evaluated for the $\mathrm{x}$-direction (by looking at the vegetation density of the two adjacent pixels in the $\mathrm{x}$-direction), for the $\mathrm{y}$-direction, and for all directions. The analysis of the conditional PDFs along the two orthogonal directions provides a useful metric to investigate spatial anisotropy. For example, the x-direction PDF conditional on neighbors having larger than average density investigates the correlation between vegetation groups and their neighbors along the $\mathrm{x}$-axis; if the $\mathrm{x}$-direction conditional PDF is shifted towards larger densities when compared to both the overall $\mathrm{PDF}$ and the y-direction conditional PDF, it is evidence that vegetated patches tend to extend along the $\mathrm{x}$-direction.

\footnotetext{
${ }^{1}$ This is a consequence of the Central Limit Theorem.
} 
Table 1. Climate, soil and vegetation properties of the system.

\begin{tabular}{|c|c|c|c|}
\hline & Niger tiger bushes & Somalia bands & Base conditions \\
\hline \multicolumn{4}{|l|}{ Soil } \\
\hline Total soil porosity, $n[-]$ & 0.4 & 0.42 & 0.4 \\
\hline Pore size distribution index, $m[-]$ & 0.4 & 0.42 & 0.4 \\
\hline Pore disconnectedness index, $c[-]$ & 8.0 & 7.8 & 8.0 \\
\hline Surface retention capacity, $h_{0}[\mathrm{~cm}]$ & 0.1 & 0.1 & 0.1 \\
\hline Saturated matric potential, $\Psi(1)[\mathrm{cm}]$ & 20 & 14 & 25 \\
\hline Depth of the water table, $Z$ [cm] & $\infty$ & $\infty$ & $\infty$ \\
\hline \multicolumn{4}{|l|}{ Climate } \\
\hline Mean storm duration, $m_{\text {tr }}$ [days] & 0.05 & 0.2 & 0.2 \\
\hline Mean time between storms, $m_{\mathrm{tb}}$ [days] & 10 & 10 & 10 \\
\hline Mean number of storms, $m_{v}[-]$ & 14.9 & 14.7 & 19.6 \\
\hline Length of the rainy season, $m_{\tau}$ [days] & 150 & 150 & 200 \\
\hline Parameter of the gamma distribution of storm depth, $k[-]$ & 0.6 & 0.6 & 0.6 \\
\hline Surface temperature $\left[{ }^{\circ} \mathrm{K}\right]$ & 300 & 300 & 300 \\
\hline Screen height temperature $\left[{ }^{\circ} \mathrm{K}\right]$ & 300 & 300 & 300 \\
\hline Specific humidity [-] & 0.01 & 0.01 & 0.01 \\
\hline Cloud fractional coverage $[-]$ & 0.0 & 0.1 & 0.1 \\
\hline Surface net radiation $\left[\mathrm{W} \mathrm{m}^{-2}\right]$ & 280 & 260 & 270 \\
\hline Mean precipitation, $m_{\mathrm{PA}}[\mathrm{cm}]$ & 56 & 16 & 40 \\
\hline \multicolumn{4}{|l|}{ Vegetation } \\
\hline Base value of transpiration efficiency, & 0.75 & 0.75 & 0.75 \\
\hline
\end{tabular}

\subsubsection{Analysis of vegetation clusters}

We arbitrarily define a cluster of vegetation as a group of adjacent pixels characterized by vegetation density larger than the domain average density. In defining a cluster, only those pixels in the von Neumann neighborhood of any given pixel are considered. For each cluster defined as above, we calculate the size, $S_{i}$, (that is, the number of pixels that compose the $i$ th cluster), the span along the $\mathrm{x}$ - and $\mathrm{y}$-directions, the shape ratio (as the ratio between the span along the $\mathrm{x}$ direction and the span along the y-direction) and the fraction of area filled (as the ratio between the cluster size and the product between the span along the two directions). We also calculate the total number of clusters present in the whole domain.

This definition of clusters allows us to compare observed and simulated vegetation clumps with the clumps resulting from a homogeneous binomial process with probability $p$ such that:

$p=\frac{\sum_{i=1}^{N_{\text {CLUSTERS }}} S_{i}}{\text { Size }_{\text {DOMAIN }}}$

We arbitrarily define a vegetation pattern as a clustered configuration whose average cluster size is higher than the 0.975 quantile of the cluster size distribution of the corresponding (through the $p$ found in Eq. 13) uniform binomial pro- cess. In addition, based on the statistical characteristics of the clumps of vegetation, we distinguish three types of pattern as follows:

- Spots: a pattern whose shape ratio is within the range 0.6-1.6.

- Bands: a pattern whose shape ratio is lower than 0.6 or higher than 1.6.

- Labyrinths: a pattern whose largest cluster is embedded in a rectangular area of at least $75 \%$ of the domain and whose fraction of area filled is less than 0.75 .

This classification implies that spots are structures whose dimensions in the $\mathrm{x}$ - and $\mathrm{y}$-directions are similar (and, thus, characterized by shape ratios close to 1 ), while bands are characterized by having a dominant dimension (either on $\mathrm{x}$ or y). Labyrinthine patterns, on the other hand, are characterized by a few big clumps of vegetation (thus the reason why we look at the areal span of the largest cluster) embedding several patches or stripes of bare soil (thus the reason why we look at the area of the cluster effectively filled with vegetation).

\subsection{Simulated patterns versus natural patterns}

Below, we present a quantitative comparison between simulated vegetation patterns and natural patterns observed in two 
African locations, namely an area of Niger near Niamey and a region of Somalia near Garoowe.

\subsubsection{Niger}

The area situated about $45 \mathrm{~km}$ south of Niamey, the capital of Niger, is known for the characteristic vegetation patterns known as tiger bushes. The average annual precipitation of the region is $56 \mathrm{~cm}$, half of which falls at an intensity higher than $35 \mathrm{~mm} \mathrm{~h}^{-1}$ and a third above $50 \mathrm{~mm} \mathrm{~h}^{-1}$ (Bromley et al., 1997). Soil is gravelly sandy loam and is highly prone to crusting in bare areas, while vegetation is concentrated in stripes of a few tens of meters wide and a few hundred meters long (Bromley et al., 1997).

The model parameters for these simulations correspond to the climatic and hydraulic characteristics of the area reported in the above literature and are shown in Table 1 (values of temperature, specific humidity and cloud coverage in the table were arbitrarily assigned in order to match the observed value of potential evapotranspiration). In addition, soil hydraulic conductivity at each pixel was calculated as a function of the vegetation density at that pixel and was set to span a range of $3 \times 10^{-7}$ to $9.5 \times 10^{-6} \mathrm{~m} \mathrm{~s}^{-1}$ for the crusted bare soil and a full canopy coverage, as suggested by field measurement (Bromley et al., 1997) and estimations from grain analysis (Casenave and Valentin, 1992). Values for the parameters of Eq. (4) through Eq. (12) are provided in Table 2. Since the above literature does not provide enough information for the estimation of all the parameters listed in the table, the remaining parameters (in particular the ones of Eq. 8-12) values were chosen within ecologically reasonable bounds (see Sect. 3.3.5) to tune the model output in order to qualitatively match the observations.

Google Earth aerial photographs of this region of Niger were used to infer field observations. A few random study areas were sampled from the vast region characterized by the presence of tiger bushes, all of which have a surface area of about $10^{5}$ square meters (see Fig. 4). The vegetation density at every pixel was estimated from the gray color levels of the digitized picture pixels mapped to the interval $M=0$ for those pixels characterized by bare soil, and $M=1$ for those characterized by full coverage. Photos, whose original resolution was of about $400 \times 400$ pixels, were then further processed in order to match the resolution of our study grid. This was done by superimposing our study grid on the original photo and averaging the fractional coverage of the set of pixels of the original photo that fell within the bounds of each pixel of our $50 \times 50$ grid.

An initial qualitative comparison between simulations and observations is presented in Fig. 4, where three original aerial photos are shown together with their digitized vegetation density maps and three sample results from our simulations. The figure shows a good qualitative agreement between natural and simulated patterns in terms of typical shape and di- mension of the vegetation structures and in the overall spatial configuration of the patterns within the study domain.

Results of several simulations exhibited a noteworthy sensitivity of the emerging patterns to changes in the spatial interaction functions and in particular to the dependence of $k_{v}$ (Eq. 9) and hydraulic conductivity on vegetation (Eq. 1). Differences between patterns in Fig. $4 \mathrm{~g}$ and $\mathrm{f}$, for example, are due to changes of about $5 \%$ in the coefficients of the Eq. (9); Fig. $4 \mathrm{~h}$ was obtained by increasing the soil conductivity in the interval corresponding to a fractional cover only in the range of 0.3 to 0.5 by about $10 \%$, keeping the overall span of the range fixed between $3 \times 10^{-7}$ to $9.5 \times 10^{-6} \mathrm{~m} \mathrm{~s}^{-1}$. The above considerations suggest that the combination of the mechanisms of run-on infiltration (mainly driven by plant feedback on soil conductivity) and facilitation (due to plants' improved efficiency in water use) is extremely important not only for the formation of patterns in the study area, but also for their shape and dimension. Thus, given that the global (i.e., climatic) conditions are favorable to pattern formation, the peculiar patterning is driven by the local feedbacks.

An analysis of the spatial distribution of water fluxes and soil moisture content is provided in Fig. 5. In particular, Fig. 5a shows the value of the average effective input at each pixel, computed as average precipitation plus the run-on and adjusted to account for the effect of roots as in Eq. (5). As shown, many areas receive an amount of water several times higher than the actual mean precipitation from the low permeable pixels located upstream, in accordance with field observation of concentration factors (ratio between the effective amount of water received and the actual precipitation) as high as 3 and 4 (Bromley et al., 1997). In addition, the higher values of groundwater runoff observable in correspondence of the vegetated patches (shown in Fig. 5b) confirm that vegetation favors the infiltration of the hillslope run-on. Taken together, the extra water input from upstream and the enhanced permeability of the more vegetated soil trigger a positive feedback for further vegetal biomass establishment, confirming that surface water redistribution due to infiltration of run-on is one of the main drivers of pattern formation, (Boaler and Hodge, 1962; Valentin et al., 1999; Casenave and Valentin, 1992). The latter result will be further investigated later in the paper. In accordance with published results (Borgogno et al., 2009), our simulation shows that the average soil moisture content is higher in the areas under vegetated patches (especially in their uphill side) than in the surrounding bare soil (Fig. 5c); this, in turn, creates suitable conditions for the sustainment and/or further development of vegetation.

As shown in Fig. 6, both observed and simulated PDFs of vegetation density are bimodal, supporting the observation that vegetation density is not normally distributed around the mean, as it would be expected if plants were spatially distributed as a homogeneous point process across the domain. Bimodality is more evident when the PDF of vegetal cover is conditioned on the neighbor pixel having a vegetation cover 
Table 2. Model parameters.

\begin{tabular}{|c|c|c|c|c|c|}
\hline & \multicolumn{3}{|c|}{ Niger tiger bushes } & \multirow{2}{*}{ Somalia bands } & \multirow{2}{*}{ Base conditions } \\
\hline & $A$ & $B$ & $C$ & & \\
\hline$a_{1}\left[\mathrm{~cm} \mathrm{~s}^{-1}\right]$ & $3 \times 10^{-5}$ & $3 \times 10^{-5}$ & $3 \times 10^{-5}$ & $9 \times 10^{-6}$ & $2 \times 10^{-5}$ \\
\hline$a_{2}\left[\mathrm{~cm} \mathrm{~s}^{-1}\right]$ & $5 \times 10^{-5}$ & $5 \times 10^{-5}$ & $5 \times 10^{-5}$ & $1 \times 10^{-5}$ & $4 \times 10^{-5}$ \\
\hline$a_{3}\left[\mathrm{~cm} \mathrm{~s}^{-1}\right]$ & $8 \times 10^{-5}$ & $7 \times 10^{-5}$ & $7 \times 10^{-5}$ & $3 \times 10^{-5}$ & $8 \times 10^{-5}$ \\
\hline$a_{4}\left[\mathrm{~cm} \mathrm{~s}^{-1}\right]$ & $3 \times 10^{-4}$ & $4 \times 10^{-4}$ & $4.5 \times 10^{-4}$ & $1 \times 10^{-4}$ & $3 \times 10^{-4}$ \\
\hline$a_{5}\left[\mathrm{~cm} \mathrm{~s}^{-1}\right]$ & $6 \times 10^{-4}$ & $6.5 \times 10^{-4}$ & $6.8 \times 10^{-4}$ & $7 \times 10^{-4}$ & $8 \times 10^{-4}$ \\
\hline$a_{6}\left[\mathrm{~cm} \mathrm{~s}^{-1}\right]$ & $8 \times 10^{-4}$ & $8 \times 10^{-4}$ & $8.5 \times 10^{-4}$ & $9 \times 10^{-4}$ & $9 \times 10^{-4}$ \\
\hline$a_{7}\left[\mathrm{~cm} \mathrm{~s}^{-1}\right]$ & $8.2 \times 10^{-4}$ & $8.2 \times 10^{-4}$ & $8.7 \times 10^{-4}$ & $9.1 \times 10^{-4}$ & $9.2 \times 10^{-4}$ \\
\hline$a_{8}\left[\mathrm{~cm} \mathrm{~s}^{-1}\right]$ & $8.4 \times 10^{-4}$ & $8.4 \times 10^{-4}$ & $8.9 \times 10^{-4}$ & $9.2 \times 10^{-4}$ & $9.4 \times 10^{-4}$ \\
\hline$a_{9}\left[\mathrm{~cm} \mathrm{~s}^{-1}\right]$ & $8.6 \times 10^{-4}$ & $8.6 \times 10^{-4}$ & $9.1 \times 10^{-4}$ & $9.3 \times 10^{-4}$ & $9.6 \times 10^{-4}$ \\
\hline$a_{10}\left[\mathrm{~cm} \mathrm{~s}^{-1}\right]$ & $8.8 \times 10^{-4}$ & $8.8 \times 10^{-4}$ & $9.3 \times 10^{-4}$ & $9.4 \times 10^{-4}$ & $9.8 \times 10^{-4}$ \\
\hline$b_{1}\left[\mathrm{~cm} \mathrm{~s}^{-1}\right]$ & $2 \times 10^{-4}$ & $2 \times 10^{-4}$ & $2 \times 10^{-4}$ & $1 \times 10^{-5}$ & $2 \times 10^{-4}$ \\
\hline$b_{2}\left[\mathrm{~cm} \mathrm{~s}^{-1}\right]$ & $3 \times 10^{-4}$ & $2 \times 10^{-4}$ & $2 \times 10^{-4}$ & $2 \times 10^{-4}$ & $4 \times 10^{-4}$ \\
\hline$b_{3}\left[\mathrm{~cm} \mathrm{~s}^{-1}\right]$ & $2.8 \times 10^{-3}$ & $3.3 \times 10^{-3}$ & $3.8 \times 10^{-3}$ & $7 \times 10^{-4}$ & $2.2 \times 10^{-3}$ \\
\hline$b_{4}\left[\mathrm{~cm} \mathrm{~s}^{-1}\right]$ & $3 \times 10^{-3}$ & $2.5 \times 10^{-3}$ & $2.3 \times 10^{-3}$ & $6 \times 10^{-3}$ & $5 \times 10^{-3}$ \\
\hline$b_{5}\left[\mathrm{~cm} \mathrm{~s}^{-1}\right]$ & $2 \times 10^{-4}$ & $1.5 \times 10^{-3}$ & $1.7 \times 10^{-3}$ & $2 \times 10^{-3}$ & $1 \times 10^{-3}$ \\
\hline$b_{6}\left[\mathrm{~cm} \mathrm{~s}^{-1}\right]$ & $2 \times 10^{-4}$ & $2 \times 10^{-4}$ & $2 \times 10^{-4}$ & $1 \times 10^{-4}$ & $2 \times 10^{-4}$ \\
\hline$b_{7}\left[\mathrm{~cm} \mathrm{~s}^{-1}\right]$ & $2 \times 10^{-4}$ & $2 \times 10^{-4}$ & $2 \times 10^{-4}$ & $1 \times 10^{-4}$ & $2 \times 10^{-4}$ \\
\hline$b_{8}\left[\mathrm{~cm} \mathrm{~s}^{-1}\right]$ & $2 \times 10^{-4}$ & $2 \times 10^{-4}$ & $2 \times 10^{-4}$ & $1 \times 10^{-4}$ & $2 \times 10^{-4}$ \\
\hline$b_{9}\left[\mathrm{~cm} \mathrm{~s}^{-1}\right]$ & $2 \times 10^{-4}$ & $2 \times 10^{-4}$ & $2 \times 10^{-4}$ & $1 \times 10^{-4}$ & $2 \times 10^{-4}$ \\
\hline$b_{10}\left[\mathrm{~cm} \mathrm{~s}^{-1}\right]$ & $2 \times 10^{-4}$ & $2 \times 10^{-4}$ & $2 \times 10^{-4}$ & $1 \times 10^{-4}$ & $2 \times 10^{-4}$ \\
\hline$\alpha_{1}$ & 0.01 & 0.01 & 0.01 & 0.03 & 0.01 \\
\hline$\alpha_{2}$ & 0.019 & 0.02 & 0.018 & 0.05 & 0.02 \\
\hline$\alpha_{3}$ & 0.03 & 0.03 & 0.03 & 0.04 & 0.03 \\
\hline$\alpha_{4}$ & 0.03 & 0.03 & 0.03 & 0.04 & 0.03 \\
\hline$\alpha_{5}$ & 0.09 & 0.09 & 0.09 & 0.08 & 0.09 \\
\hline$\xi_{R}$ & 1.5 & 1.5 & 1.5 & 1.25 & 1.5 \\
\hline
\end{tabular}

A)

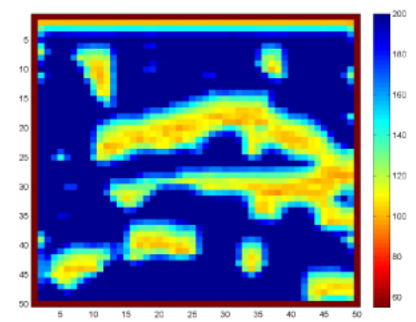

B)

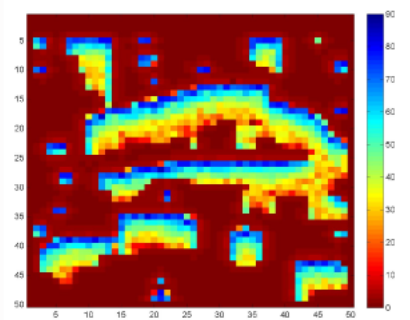

C)

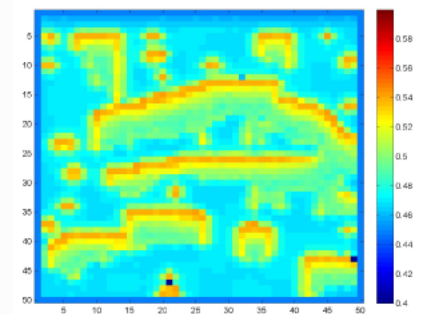

Fig. 5. (A) Ratio between the effective amount of water received and annual precipitation for the simulated pattern in Fig. 4g. (B) Long-term groundwater runoff on the study domain for the simulated pattern in Fig. 4g. (C) Long-term soil moisture content during the rainy season on the study domain for the simulated pattern in Fig. $4 \mathrm{~g}$.

higher than the domain average. The latter suggests that the vegetal density of each pixel is more likely to be higher than the average, providing that it is in the neighborhood of a pixel whose cover is also higher than the spatial average. The opposite is true when the condition is on the neighbor pixel having a density that is lower than the average. A slight prevalence of structures in the $\mathrm{x}$-direction (perpendicular to the domain slope) is apparent from the analysis of the conditional PDFs for the pattern under analysis. This is apparent from both the qualitative observation of natural and simulated structures (Fig. 4a, d and g) and from the analysis of the directional conditional PDF in Fig. 6. Looking at the interval $0<M<0.1$, in fact, the PDF of the vegetal density of pixels, being in the $\mathrm{x}$-direction neighborhood of a pixel with vegetal density higher than the domain average, shows a lower density than the y-directional conditional 
A)

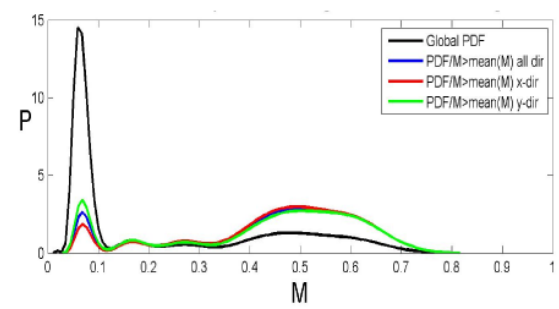

C)

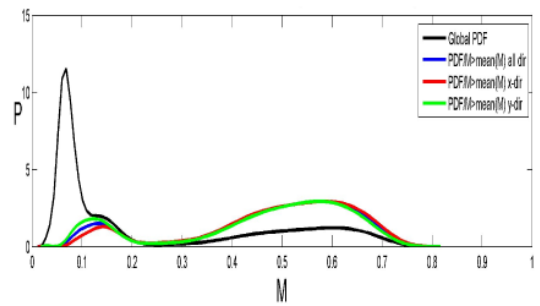

B)

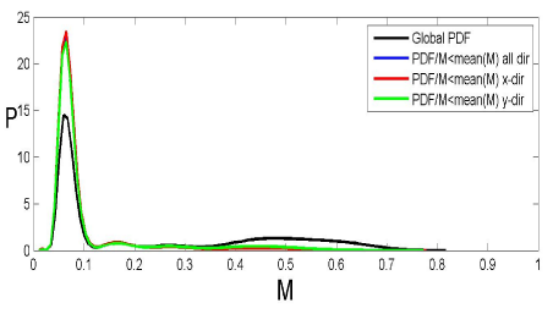

D)

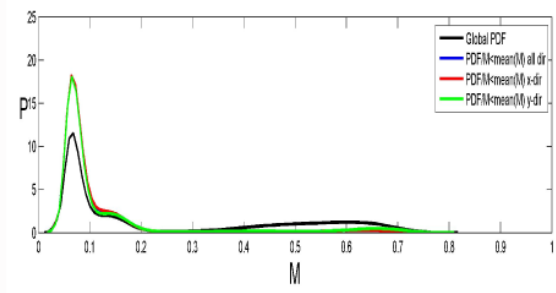

Fig. 6. Global and conditioned PDF analysis of the Niger patterns. Panels (A/B): natural, and (C/D): simulated vegetation; panels (A/C): density higher than global average, and (B/D): density lower than global average. (A) PDFs of vegetation density for the natural pattern shown in Fig. 4a (as digitized in Fig. 4d): global PDF (black line) and PDFs of vegetation density conditioned on having a neighbor pixel with vegetation density higher than the global average for all directions (blue line), x-direction (red line) and y-direction (green line). (B) PDFs of vegetation density for the natural pattern shown in Fig. 4a (as digitized in Fig. 4d): global PDF (black line) and PDFs of vegetation density conditioned on having a neighbor pixel with vegetation density lower than the global average for all directions (blue line), $\mathrm{x}$-direction (red line) and y-direction (green line). (C) PDFs of vegetation density for the simulated pattern shown in Fig. 4g: global PDF (black line) and PDFs of vegetation density conditioned on having a neighbor pixel with vegetation density higher than the global average for all directions (blue line), x-direction (red line) and y-direction (green line). (D) PDFs of vegetation density for the simulated pattern shown in Fig. 4g: global PDF (black line) and PDFs of vegetation density conditioned on having a neighbor pixel with vegetation density lower than the global average for all directions (blue line), $\mathrm{x}$-direction (red line) and y-direction (green line).

A)
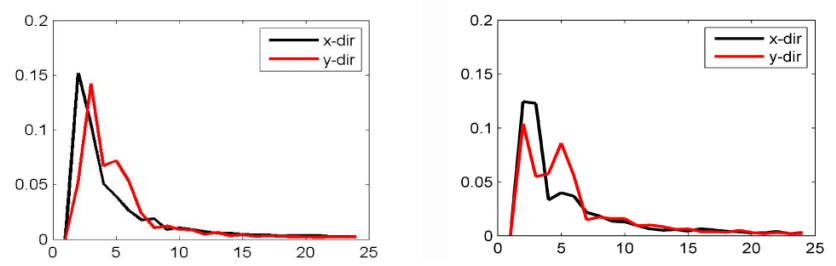

Fig. 7. Power spectral densities of the vegetation density field; frequencies (in the horizontal axis) are expressed in terms of number of wavelengths present within the domain length and width. (A) Average of the 1-D power spectral densities of the vegetation density for the natural pattern shown in Fig. 4a along the x-direction (black line) and y-direction (red line). (B) Average of the 1-D power spectral densities of the vegetation density for the simulated pattern shown in Fig. $4 \mathrm{~g}$ along the $\mathrm{x}$-direction (black line) and $\mathrm{y}$-direction (red line).

PDF. Conversely, for the same interval of fractional coverage, higher density is shown for the PDF of the vegetal density of pixels being in the $\mathrm{x}$-direction neighborhood of a pixel with vegetal density lower than the domain average.
A)
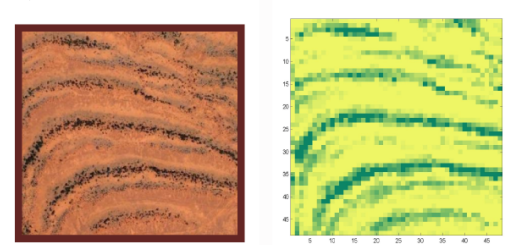

C)

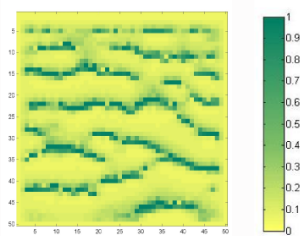

Fig. 8. (A) Aerial photograph of a natural vegetation pattern in Somalia $\left(7^{\circ} 43^{\prime} \mathrm{N}, 48^{\circ} 02^{\prime} \mathrm{E}\right)$; (B) Digitized representation of the vegetation cover of the natural pattern in (A). (C) Sample simulated pattern.

Spectral analysis of the vegetation density field $M(\mathrm{x}, \mathrm{y})$ is provided for both natural and simulated patterns in Fig. 7. Average of the one-dimensional spectral densities, evaluated for both the $\mathrm{x}$-direction and the $\mathrm{y}$-direction, are provided along with the two-dimensional power spectrum. Peak spectral densities appear in correspondence with the lower frequencies, capturing the presence of the large-scale structures. In agreement with the analysis of the directional PDFs, spectral analysis confirms the presence of a slight anisotropy in the pattern shape, for both the natural and the simulated case, 
by detecting a prevalence of structures recurring at frequencies between 2 and 6 (that is, 2 to 6 structures per domain length) along the y-direction.

The analyses of the characteristics of the vegetation clusters of both natural and simulated vegetation fields, whose results are reported in Table 3, show that two out of three natural patterns (Fig. $4 \mathrm{~d}$ and e) fall within our definition of spots, while the pattern in Fig. 4f is labyrinthine. Simulated patterns, however, are all spotted, even though the one shown in Fig. 4i, whose largest cluster covers the $68 \%$ of the domain, almost meets the requirement for being classified as labyrinthine.

\subsubsection{Somalia}

Vegetation stripes are a widespread occurrence on the Somaliland plateau (Boaler and Hodge, 1962) and in the Puntland area (Borgogno et al., 2009). The area is semi-desert and characterized by an arid climate with precipitation highly variable in space and time (Muchiri, 2007). The area selected is located about $30 \mathrm{~km}$ west of Garoowe, the administrative capital of the Puntland region of Somalia and is characterized by annual precipitation ranging between 10 and $20 \mathrm{~cm} \mathrm{yr}^{-1}$, mostly occurring in the period of May-September (Muchiri, 2007). Dominant soils are Gypsisol and Calcisol (Venema, 2007), according to the FAO definition, and are characterized by hydraulic conductivity that can be as low as a few $\mathrm{cm} /$ day, but can span two orders of magnitude. These kinds of soil are also very susceptible to crusting and cracking (Driessen et al., 2001).

A study area of about $5 \times 10^{5} \mathrm{~m}^{2}$, located at $7^{\circ} 43^{\prime} \mathrm{N}$, $48^{\circ} 02^{\prime} \mathrm{E}$ and characterized by the presence of vegetation stripes was selected from the Puntland area near Garoowe. In comparison to the Niger case, the climate is drier (precipitation being less than one third that of the Niger case) and patterns - in this case well-defined stripes - occur at a slightly larger scale. In accordance with the climatic and soil information reported above, the simulations of the area used the parameters shown in Tables 1 and 2. Results of the simulations were compared to the observed patterns, as shown in Fig. 8 through Fig. 10.

As done for the case of the Niger tiger bushes, a sample aerial photograph was processed in order to obtain estimates of the vegetal density at a $50 \times 50$ study grid, and compared with the simulated results (Fig. 8). The comparison shows a good qualitative match between observed and simulated vegetal spatial configurations. Dimensions and shape of the bands and of the inter-band gaps are similar, although the vegetation structures emerging from the simulations look slightly sharper than the observed ones. In addition, orientation of the bands perpendicular to the slope is clearer in the simulations than in the observations. This is because, although the direction of the natural and simulated slope was set to coincide, the natural topography presented some irregularities, and the simulations were performed on a reg- ular slope. Nevertheless, both in the observed and simulated case, the configuration of vegetal density is characterized by stripes of vegetation a few tens of meters wide downslope from and extending across the entire study domain.

Compared to the previously analyzed case of the Niger tiger bushes, the directionality of the Somaliland plateau vegetation patterns is more noticeable, both in the observations and in the simulations. Moreover, the total amount of vegetation (integrated across the domain) is smaller than in the case of the Niger tiger bushes, as easily noticeable from the comparison of the PDFs of the vegetal density shown in Figs. 6 and 9. Analysis of the PDFs in Fig. 9 also shows that the presence of multiple modes is less evident here than in the case of the Niger tiger bushes, when the unconditioned PDF of the vegetation density is considered. However, multiple modes become apparent in the conditional PDFs of both the observed and the simulated vegetation fields, especially with respect to the PDF in the $\mathrm{x}$-direction conditioned on neighbor density higher than the domain average. As observed in the case of the Niger patterns, the presence of multiple modes in the conditional PDFs and, in general, the fact that the conditional PDFs look different than the unconditional PDF, implies that the vegetation at a given pixel has an impact on the vegetation distribution of its neighborhood. In particular, the probability of finding a pixel whose fractional coverage is higher than the average is greater in a neighborhood of pixels that are themselves characterized by above-average density, supporting the observation that vegetation tends to form clumps rather than being distributed completely randomly in space. In addition, the PDF conditioned on $\mathrm{x}$ direction neighbor density higher than the domain average, shows higher density for values of vegetation in the upper end of the domain interval than the other (conditional and unconditional) PDFs; this confirms the prevalence of stripes of vegetation in the $\mathrm{x}$-direction itself, that is, perpendicular to the domain slope.

Spectral analysis (Fig. 10) supports the conclusions drawn from both the qualitative analysis and the analysis of the PDFs of the vegetation density, showing the presence of frequencies between 5 and 10 cycles along the domain only along one direction. However, comparisons between the spectral densities of the observed and simulated vegetation field also imply that the prevalence of stripes perpendicular to the main slope is higher in the simulated field than the observed. Since the only anisotropic effect present in our model is topographic (through the surface runoff production and run-on infiltration), and the shape of the natural pattern seems to indicate that the governing mechanism of pattern formation is topographic and gravitational, we attribute the discrepancy between observations and simulations to the irregularities of the natural topography of the observed area with respect to the regular slope used in the simulated domain.

Statistical analysis of the vegetation clusters, whose results are reported in Table 3, show that both natural and simulated patterns are banded. 
Table 3. Cluster analysis for natural and simulated patterns in Niger and Somalia.

\begin{tabular}{|c|c|c|c|c|c|c|c|c|}
\hline \multicolumn{6}{|c|}{ Niger } & \multicolumn{3}{|c|}{ Somalia } \\
\hline & Natural & & & Simulat & & & Natural & Simulated \\
\hline Figure & $4 d$ & $4 \mathrm{e}$ & $4 \mathrm{f}$ & $4 \mathrm{~g}$ & $4 \mathrm{~h}$ & $4 \mathrm{i}$ & $15 b$ & $15 \mathrm{c}$ \\
\hline Number of clusters & 21 & 20 & 29 & 22 & 30 & 14 & 49 & 24 \\
\hline Average cluster size & 42 & 38 & 38 & 37 & 32 & 90 & 15 & 28 \\
\hline $\begin{array}{l}\text { Range } 0.025-0.975 \text { quantile of } \\
\text { cluster size for binomial process }\end{array}$ & {$[1-18]$} & {$[1-12]$} & {$[1-35]$} & {$[1-14]$} & {$[1-24]$} & {$[1-74]$} & {$[1-12]$} & [1-9] \\
\hline Shape ratio & 1.4 & 1.5 & 1.1 & 1.2 & 1.4 & 1.2 & 1.9 & 2.6 \\
\hline $\begin{array}{l}\text { Percentage of domain filled by the } \\
\text { largest cluster }\end{array}$ & 0.35 & 0.34 & 0.78 & 0.57 & 0.18 & 0.68 & 0.36 & 0.15 \\
\hline $\begin{array}{l}\text { Fraction of area of the largest cluster } \\
\text { filled with vegetation }\end{array}$ & 0.51 & 0.35 & 0.37 & 0.42 & 0.63 & 0.44 & 0.21 & 0.23 \\
\hline Cluster type & Spots & Spots & Labyr & Spots & Spots & Spots & Bands & Bands \\
\hline
\end{tabular}

Table 4. Statistical analysis of the vegetation clusters for the fields of vegetation obtained with hydraulic conductivity functions, modified from the base conditions through Eq. (14).

\begin{tabular}{|c|c|c|c|c|c|c|c|c|c|}
\hline Coefficient $A$ & -0.4 & -0.3 & -0.2 & -0.1 & 0 & 0.1 & 0.2 & 0.3 & 0.4 \\
\hline Number of clusters & 11 & 19 & 112 & 109 & 61 & 54 & 80 & 61 & 90 \\
\hline $\begin{array}{l}\text { Range } 0.025-0.975 \text { quantile of } \\
\text { cluster size for binomial process }\end{array}$ & {$[1-24]$} & [1-20] & [1-26] & {$[1-25]$} & [1-21] & {$[1-25]$} & {$[1-22]$} & {$[1-23]$} & {$[1-25]$} \\
\hline Shape ratio & 1.2 & 1.5 & 7.0 & 9.1 & 5.9 & 5.0 & 3.7 & 6.2 & 4.9 \\
\hline $\begin{array}{l}\text { Percentage of domain filled by } \\
\text { the largest cluster }\end{array}$ & 0.56 & 0.36 & 0.40 & 0.43 & 0.24 & 0.35 & 0.28 & 0.36 & 0.29 \\
\hline $\begin{array}{l}\text { Fraction of area of the largest } \\
\text { cluster filled with vegetation }\end{array}$ & 0.27 & 0.43 & 0.47 & 0.50 & 0.48 & 0.32 & 0.38 & 0.36 & 0.41 \\
\hline
\end{tabular}

\subsection{Analysis of the hypothesized pattern-promoting dynamics}

\subsubsection{Impact of climate forcings}

In order to explore the effect of the climate forcing on pattern formation and spatial characteristics of the vegetation distribution, the system was simulated by varying the climatic forcing, while keeping everything else fixed. All the inputs for these simulations are reported in the column titled "base conditions" of Tables 1 and 2. The soil-climatic conditions referred to here as base conditions are arbitrary and not representative of a specific site. We investigated the impact of two climatic components: mean annual precipitation and mean annual net radiation (through the impact that the latter has on potential rates of evapotranspiration).

Simulations showed that the shape and the presence of patterns at the hillslope scale depend not only on the mean annual precipitation but also on the characteristics of the rainy events (mean storm duration, mean time between storms, mean storm intensity, etc.). Below, we focus our analysis only on the dependence on the mean annual precipitation.
Figure 11 shows the result of the statistical analysis of the vegetation fields obtained for a range of mean annual precipitation of 26 to $85 \mathrm{~cm}$ (the range was selected to be wide enough to detect the transition from bare hillslope to patterns and to densely vegetated hillslope without patterns).The variation in mean annual precipitation was achieved by varying the mean storm duration only and leaving the mean storm intensity unchanged. All the other parameters of the model, both climatic and hydraulic, were kept at the values set for the "base conditions". Figure 11a shows that patterns of vegetation start to emerge for mean annual precipitation higher than $32 \mathrm{~cm}$ and that cease to exist when mean precipitation approaches $60 \mathrm{~cm}$ per year. Nearly all the patterned fields are banded, as shown in Fig. 11b, and none presents labyrinthine characteristics, as shown in Fig. 11c and d. Three sample fields obtained for annual precipitation of 70, 48 and $32 \mathrm{~cm}$ per year are presented in Fig. 12 for a visual interpretation of the transition from undistinguishable patterns to selforganized structures.

Net radiation has the opposite effect, that is, patterns start to emerge as the net radiation increases, everything else being equal (Figs. 13 and 14). Patterns emerge as net radiation exceeds $230 \mathrm{~W} \mathrm{~m}^{-2}$. As net radiation increases, and, therefore, 
A)

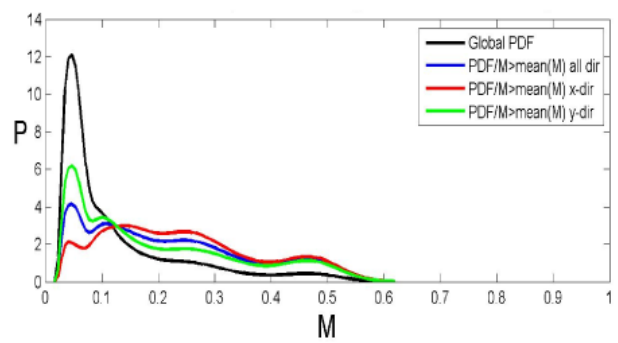

C)

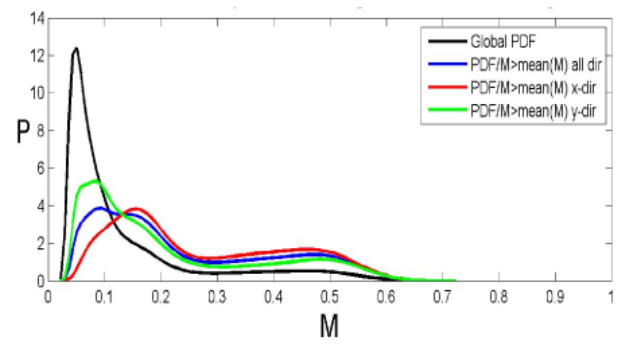

B)

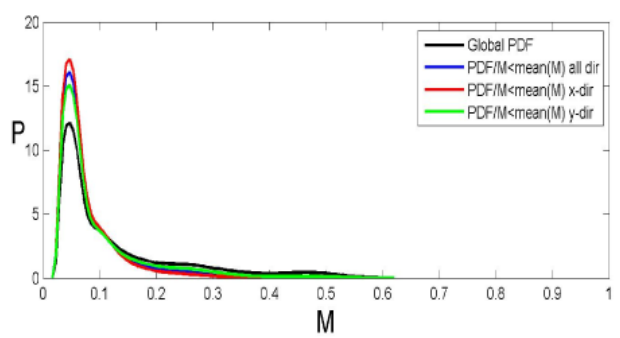

D)

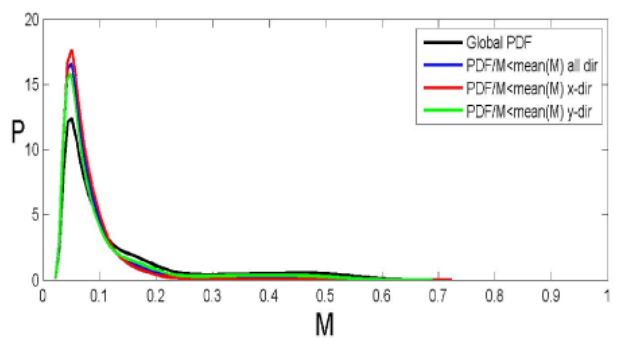

Fig. 9. Global and conditioned PDF analysis of the Somalia patterns. Panels (A/B): natural, and (C/D): simulated vegetation; panels (A/C): density higher than global average, and (B/D): density lower than global average. (A) PDFs of vegetation density for the natural pattern shown in Fig. 8a (as digitized in Fig. 8b): global PDF (black line) and PDFs of vegetation density conditioned on having a neighbor pixel with vegetation density higher than the global average for all directions (blue line), x-direction (red line) and y-direction (green line). (B) PDFs of vegetation density for the natural pattern shown in Fig. 8a (as digitized in Fig. 8b): global PDF (black line) and PDFs of vegetation density conditioned on having a neighbor pixel with vegetation density lower than the global average for all directions (blue line), $\mathrm{x}$-direction (red line) and y-direction (green line). (C) PDFs of vegetation density for the simulated pattern shown in Fig. 8c: global PDF (black line) and PDFs of vegetation density conditioned on having a neighbor pixel with vegetation density higher than the global average for all directions (blue line), x-direction (red line) and y-direction (green line). (D) PDFs of vegetation density for the simulated pattern shown in Fig. 8a: global PDF (black line) and PDFs of vegetation density conditioned on having a neighbor pixel with vegetation density lower than the global average for all directions (blue line), $\mathrm{x}$-direction (red line) and y-direction (green line).

A)
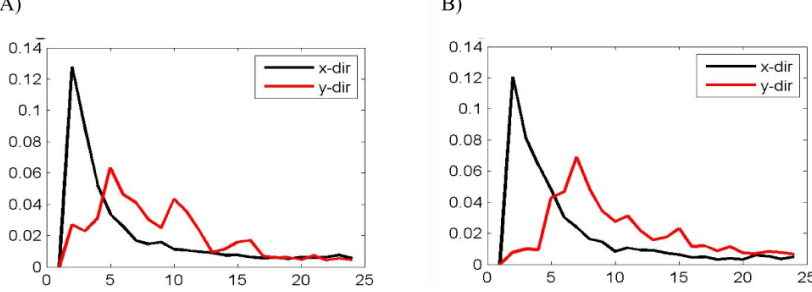

Fig. 10. Power spectral densities of the vegetation density field; frequencies (in the horizontal axis) are expressed in terms of number of wavelengths present within the domain length and width. (A) Average of the 1-D power spectral densities of the vegetation density for the natural pattern shown in Fig. 4a along the x-direction (black line) and y-direction (red line). (B) Average of the 1-D power spectral densities of the vegetation density for the simulated pattern shown in Fig. 8c along the $\mathrm{x}$-direction (black line) and y-direction (red line). climatic conditions become more arid, clumps of vegetation decrease in number and increase in average size.

Neither precipitation nor net radiation is, by itself, sufficient to characterize the aridity of a given climate. In order to incorporate both parameters, therefore, like Eagleson (1978) we define potential humidity, $\mathrm{PH}$, as the ratio between the annual precipitation and the domain-averaged annual potential evapotranspiration, and estimate the relationship between $\mathrm{PH}$ and the spatial characteristics of the simulated vegetation fields. Results of the statistical analysis of the vegetation clusters as a function of $\mathrm{PH}$ are reported in Fig. 15. We notice that vegetation patterns arise for $\mathrm{PH}$ values between 0.2 and 0.3 and that, for the other climatic and hydraulic conditions characterizing this set of simulations, all patterns are banded.

In general, the analysis of Figs. 11, 13, and 15 supports the observation that patterns arise in arid areas, that is, in water-limited environments, agreeing with all the available literature on the topic. For wetter climates, in fact, (as occurs here for PH higher than 0.3 ) the vertical water input is 
A)

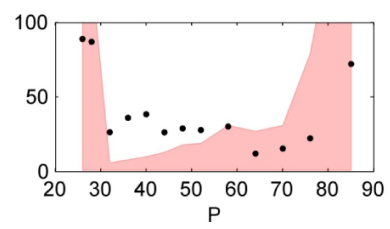

C)

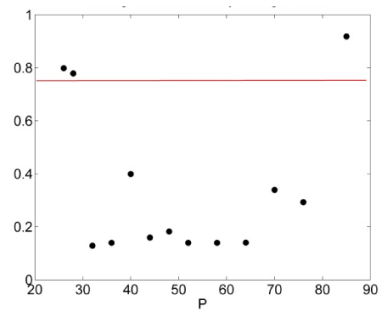

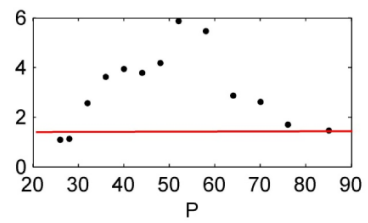

D)

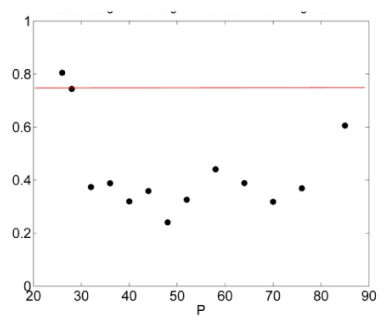

Fig. 11. Statistical analysis of the vegetation clusters for the fields of vegetation obtained by varying annual precipitation. (A) Mean size of clusters and range $0.025-0.975$ quantile of the mean cluster size corresponding to a binomial process with the same percentage cover. (B) Mean ratio between the span in the x-direction and the span in the y-direction of each cluster; red line represents the boundary between spots and bands. (C) Percentage of the domain filled by the largest cluster; red line represents the minimum fraction of area filled for the field to be considered labyrinthine. (D) Fraction of area of the largest cluster filled by vegetation; red line represents the maximum fraction of the largest cluster that can be filled for the field to be considered labyrinthine.

A)

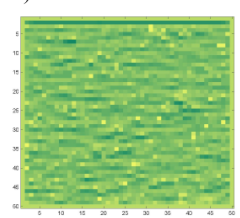

B)

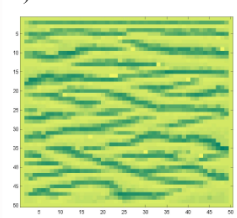

C)

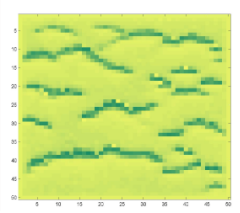

A)

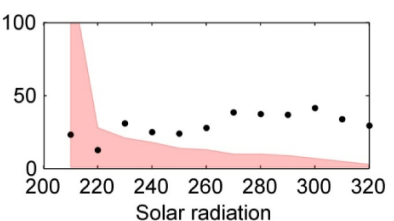

C)

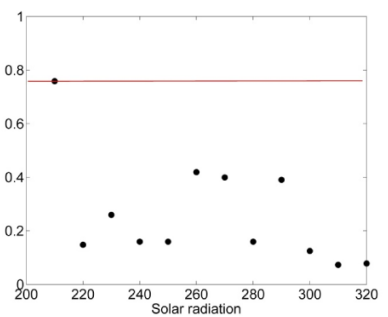

B)

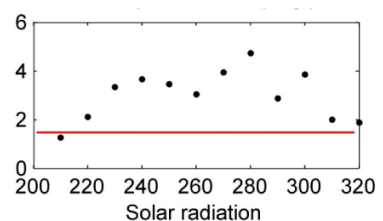

D)

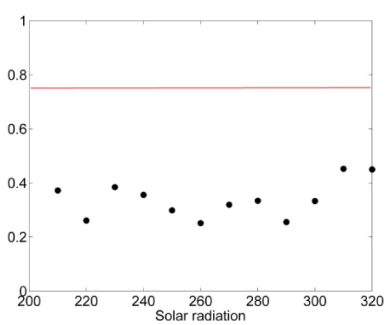

Fig. 13. Statistical analysis of the vegetation clusters for the fields of vegetation obtained by varying average net radiation. (A) Mean size of clusters and range 0.025-0.975 quantile of the mean cluster size corresponding to a binomial process with the same percentage cover. (B) Mean ratio between the span in the x-direction and the span in the $y$-direction of each cluster; red line represents the boundary between spots and bands. (C) Percentage of the domain filled by the largest cluster; red line represents the minimum fraction of area filled for the field to be considered labyrinthine. (D) Fraction of area of the largest cluster filled by vegetation; red line represents the maximum fraction of the largest cluster that can be filled for the field to be considered labyrinthine.

A)

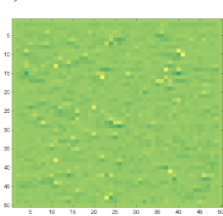

B)

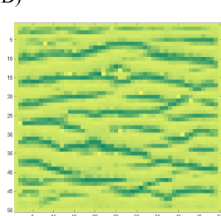

C)

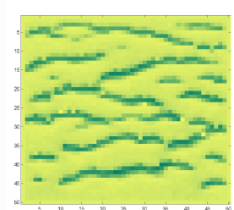

Fig. 12. Effect of precipitation on pattern formation. (A) $P=$ $32 \mathrm{~cm} \mathrm{yr}^{-1}$; (B) $P=48 \mathrm{~cm} \mathrm{yr}^{-1}$; (C) $P=70 \mathrm{~cm} \mathrm{yr}^{-1}$.

enough to support a substantial amount of vegetation even in the absence of surface water redistribution or mechanisms of facilitation/competition. When this occurs, the impact of those dynamics of surface water redistribution and facilitation/competition becomes comparatively less important (that is, the lateral interactions are overpowered by the vertical water and energy fluxes) and does not induce the emergence of recognizable patterns. As the conditions become more arid, that is, for lower $\mathrm{PH}$ values (in our simulations for $0.2<\mathrm{PH}<0.3$ ), the water input from lateral redistribution becomes determinant for the amount of vegetation that establishes at each pixel; moreover, the benefits of the facilitation mechanisms that exist in the neighborhood of vegetated pixels become (comparatively to more humid conditions) more significant and, thus, promote vegetation rearrangement and pattern formation. For the lowest $\mathrm{PH}$ values,

Fig. 14. Effect of net radiation on pattern formation. (A) Net radiation $=210 \mathrm{~W} \mathrm{~m}^{-2}$; (B) Net radiation $=250 \mathrm{~W} \mathrm{~m}^{-2}$; (C) Net radiation $=290 \mathrm{~W} \mathrm{~m}^{-2}$.

that is, for the most arid conditions explored in this analysis, the climate conditions are so adverse to vegetation establishment that the study hillslope tends to be too scarcely vegetated for the facilitation/competition dynamics to take place, ultimately resulting in the absence of patterns.

\subsubsection{Temporal dynamics}

Some vegetation patterns tend to migrate uphill (Worral, 1959, 1960; Valentin et al., 1999; Sherrat, 2005). Although we do not explicitly include a time description of the system evolution, our modeling approach has an implied time evolution, therefore allowing inferences about the development of the system over time from its evolution through the numerical iterative process. Once a pattern is established, in fact, the 
A)

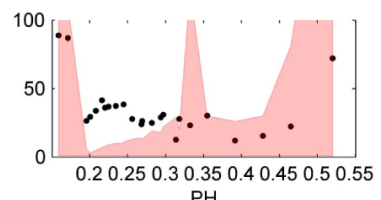

$\mathrm{PH}$

C)

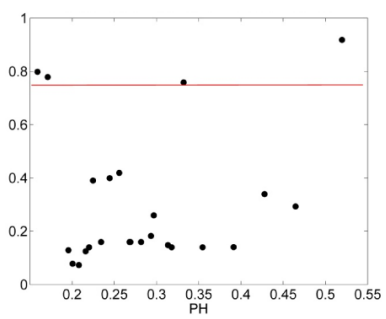

B)

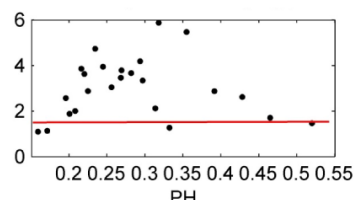

$\mathrm{PH}$

D)

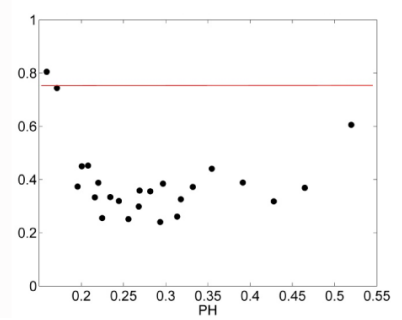

Fig. 15. Statistical analysis of the vegetation clusters for the fields of vegetation as a function of the ratio between annual precipitation and annual potential evapotranspiration. (A) Mean size of clusters and range $0.025-0.975$ quantile of the mean cluster size corresponding to a binomial process with the same percentage cover. (B) Mean ratio between the span in the $\mathrm{x}$-direction and the span in the $y$-direction of each cluster; red line represents the boundary between spots and bands. (C) Percentage of the domain filled by the largest cluster; red line represents the minimum fraction of area filled for the field to be considered labyrinthine. (D) Fraction of area of the largest cluster filled by vegetation; red line represents the maximum fraction of the largest cluster that can be filled for the field to be considered labyrinthine.

vegetal density at each pixel can either remain fixed (or not change significantly in between simulation steps), or it can undergo changes that - although significant at the pixel level - do not alter the macroscopic structure of the pattern itself, as in the case of vegetation structures that migrate across the domain.

In order to analyze this effect, we tracked the evolution of the vegetation field corresponding to the base conditions through different iteration steps, as shown in Fig. 16. The figure shows the vegetation field at the 45th, 50th, 55th and 60th iteration step of our simulation procedure on a domain sloped from top to bottom in the figure. In this regard, we emphasize the fact that slope is only included to determine the direction of the surface run-on, as outlined in Eq. (3). It is evident that the patterns have already been established by the 45 th step of the iteration, shown in Fig. 16a, and that the stripes migrate uphill as the simulation progresses. In addition to the migration, we notice that most bands are convex downslope, in accordance with many observations (Worral, 1959). As reported in the cited literature, pattern migration is induced by the effect of surface run-on ponding and infiltration in the uphill part of the stripes, which in turn creates a favorable opportunity for uphill expansion or migration of the vegetation. This claim is supported by the analysis of the spatial distribution of soil moisture and water fluxes (not shown),

confirming the presence of wetter soils and higher infiltration in the uphill portion of the vegetation bands, which as the simulation progresses, creates a favorable environment for further vegetation establishment and a positive feedback for the uphill expansion of the vegetation clumps. The drier conditions observed in the downhill portion of the bands, created by the fact that most of the available run-on has already infiltrated, result in the creation of adverse conditions that inhibit vegetation establishment.

However, a pattern migration was not observed in all the simulated cases. Several simulations (not shown) developed patterns that, once established, did not exhibit any tendency to migrate from their original location. This is attributed to the predominance of the local inhibition dynamics present in the uphill portion of vegetation clusters. In those cases, in fact, it has been observed for the pixels immediately uphill of a clump of vegetation that the inhibition effect, due to the terms in Eqs. (10) and (11) (which reflect the effect of soil erosion due to the surface runoff), overpower the facilitation due to the presence of vegetation immediately downhill. In the real world, the strength of those inhibiting/facilitating factors would be determined by: overland flow velocity, soil texture, nutrients content, amount of litter produced by the vegetation, characteristics of the vegetation crown its impact on light exposure, etc. All those factors are not explicitly taken into account here, but are implicitly considered in the magnitude of coefficients in Eq. (8) through Eq. (11) and in their relative strength with respect to the processes directly impacting the water fluxes.

\subsubsection{Impact of hydraulic conductivity}

Regions where vegetation patterns occur are characterized by soils whose permeability is highly variable in space, being higher in areas of vegetated soil and lower where the soil is bare (HilleRisLambers et al., 2001; Bromley et al., 1997; Valentin et al., 1999; Boaler and Hodge, 1962; Saco et al., 2007). We account for the effect of plants on the permeability of the soil by expressing hydraulic conductivity as a function of vegetation density. In order to explore the impact that a vegetation-dependent hydraulic conductivity has on pattern formation, we consider the following three cases: (1) hydraulic conductivity determined at each pixel as a function of vegetation according to Eq. (1) (base conditions); (2) spatially uniform hydraulic conductivity equal to the spatial average corresponding to the base conditions; and (3) hydraulic conductivity at each pixel, randomly sampled from a uniform distribution spanning the same range of hydraulic conductivities as in the base conditions. The first case encodes the vegetation-hydraulic conductivity feedback, the last two cases do not.

As shown in Fig. 17a, no patterns emerged in the case of constant hydraulic conductivity. Similarly, no well-defined structures emerged in the case of spatially random hydraulic conductivity. In this latter case, the spatial variability of the 

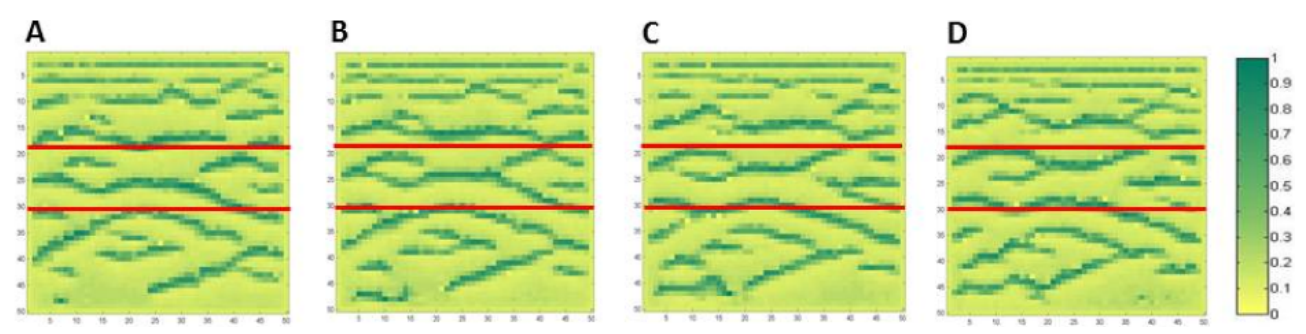

Fig. 16. Pattern migration. The evolution of a pattern is tracked through iteration steps (use red lines as reference): (A) 45; (B) 50; (C) 55; (D) 60 .

A)
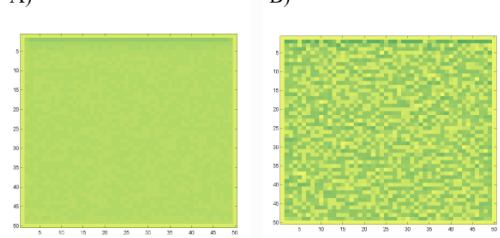

C)

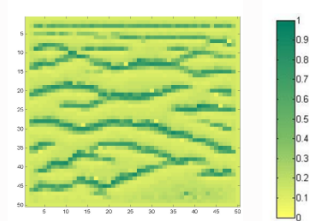

Fig. 17. Effect of hydraulic conductivity on pattern formation. (A) Uniform hydraulic conductivity. (B) Hydraulic conductivity randomly variable in space. (C) Hydraulic conductivity variable in space as a function of vegetation density.

resulting vegetation density is higher than in the case with constant conductivity (as apparent in Fig. 17b) but with a distribution that does not present the bimodality and asymmetry of distributions with well-defined patterns (Fig. 22). In particular, the vegetation that arises in the case of spatially random hydraulic conductivity (Fig. 17b) traces the spatial distribution of the hydraulic conductivity itself (pixels with higher conductivity soils are more vegetated than those where the soil is less permeable). In both cases in Fig. 17a and $b$, the absence of feedback between vegetation and the hydraulic properties of the soil prevents well-defined patterns from emerging, even when all the other spatial effects are in play. This result indicates that the ability of vegetation to affect the soil properties is crucial to promote water redistribution via runoff production and infiltration. Although not shown, in fact, no patterns were observed in the hypothetical case of perfectly horizontal hillslope, simulating the extreme condition of absence of redistribution of surface run-on. This, along with the previous findings, individuates in the mechanisms of surface runoff production and surface run-on infiltration the primary drivers of the phenomenon for a given set of climatic conditions.

Once it has been established that dependence of hydraulic conductivity on vegetation density is essential to promote the water flux redistribution necessary to produce patterns, we investigate the role played by the shape of the function in Eq. (1) in the ultimate vegetation configuration. To this purpose, we simulate the system with a set of alternative functions of the type in Eq. (1). Those equations were obtained from the base condition equation (whose parameters are re-

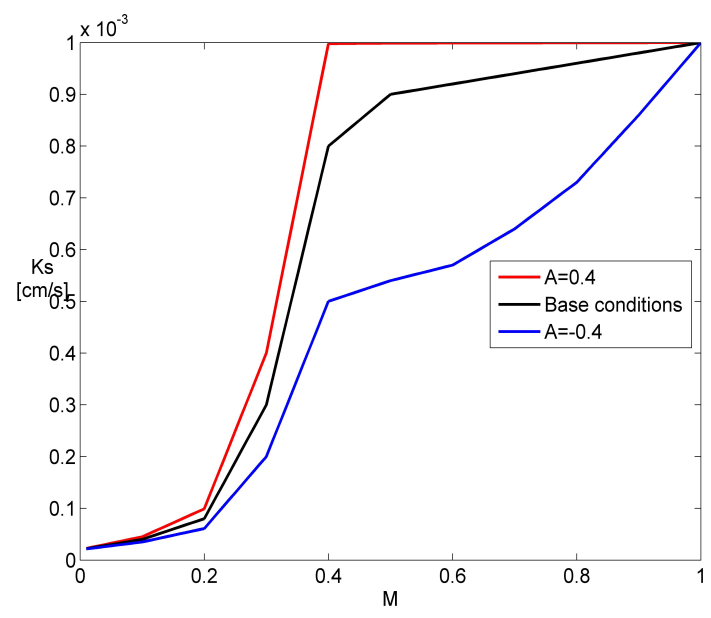

Fig. 18. Hydraulic conductivity functions for the base conditions (black), compared with the functions obtained through Eq. (14) with coefficients $A=0.4$ (red) and $A=-0.4$ (blue).

ported in Table 2) by using the following transformation:

$$
\begin{aligned}
& \left\{K_{S}(M)\right\}_{i}=\max \left\{\mathrm{lb}, \min \left\{\mathrm{ub},\left[1+A_{i}\right.\right.\right. \\
& \left.\left.\cdot \sin (\pi \cdot M)] \cdot\left\{K_{S}(M)\right\}_{\text {Base Conditions }}\right\}\right\},
\end{aligned}
$$

where $\left\{K_{S}(M)\right\}_{i}$ is the hydraulic conductivity function of simulation $i, \mathrm{lb}$ and $\mathrm{ub}$ are the lower and upper bound of the base condition function, respectively, and $A_{i}$ represents a scaling factor. Such formulation allows us to simulate the system using hydraulic conductivity functions that span the same range as that of the base condition, while varying the shape of those functions, as shown in Fig. 18.

Eight simulations were performed using hydraulic conductivity functions obtained through Eq. (14), with coefficients $A$ equal to: $-0.4,-0.3,-0.2,-0.1,0.1,0.2,0.3$ and 0.4 . The statistical analysis of the vegetation clusters, shown in Table 4, shows the impact that the shape of the hydraulic conductivity function has on the spatial configuration of the vegetation. As shown in Fig. 18, patterns emerge only for $A \geq-0.3$, and are banded only for $A \geq-0.2(A=-0.3$ produces a spotted configuration according to our cluster classification criteria). No labyrinthine configurations were found with the analyzed set of conditions (the model, however, is able to reproduce patterns for other combinations of 
A)

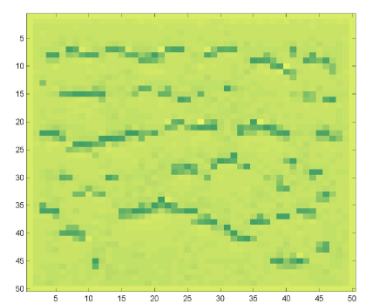

B)

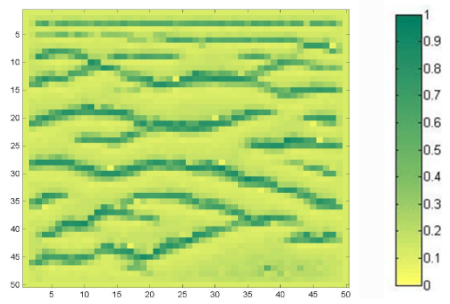

Fig. 19. Effect of local plants' interactions on pattern formation. (A) No interactions. (B) Spatially variable (function of neighbors' vegetation and surface runoff) interactions.

climatic and soil conditions, not included in this paper). As shown in Fig. 18, the functions obtained through Eq. (14) are not significantly different from that of the base conditions, even in the two limit cases of $A=-0.4$ and $A=0.4$. Nevertheless, even these small differences have a strong impact on the system response. For example, in the case of $A=-0.4$, which leads to no patterns, the PDF of the resulting vegetation density distribution has modes at $M \approx 0.1$ (bare soil patches) and $M \approx 0.45$ (vegetated clumps). Those two values of $M$ correspond to the range for which the slope of the hydraulic conductivity function of the base conditions (i.e., $A=0$ ) is higher than for the case $A=-0.4$. In the base conditions simulation, this large function gradient allows areas with $M \approx 0.4$ to be sufficiently permeable to favor water infiltration and further vegetation establishment, triggering the positive feedback which ultimately promotes pattern formation. In the case of $A=-0.4$, instead, the soil permeability required to favor run-on infiltration would be reached in areas with vegetal cover $M>0.7$, which is too high to be sustainable, given the climatic and the hydraulic properties of the system. Other simulations performed on different sets of climatic and hydraulic conditions support this finding and indicate that each set of climatic and hydraulic conditions requires the hydraulic conductivity function to have a particular shape for the vegetation configuration to be patterned. Specifically, the hydraulic conductivity function must be such that: (1) the permeability for low vegetated areas (e.g., $M<0.2$ ) promotes the formation of surface runoff without allowing further establishment of vegetation (and, thus, positive feedback on permeability) and (2) the vegetated areas (e.g., $M>0.4$ ) are permeable enough to allow run-on infiltration and sustain (for the given climatic conditions) their vegetal coverage and/or promote further vegetation establishment.
A)

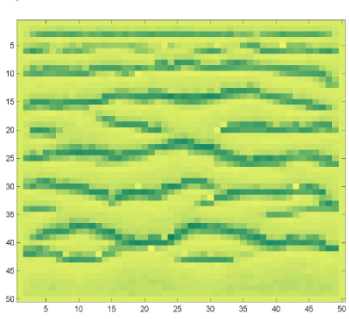

B)

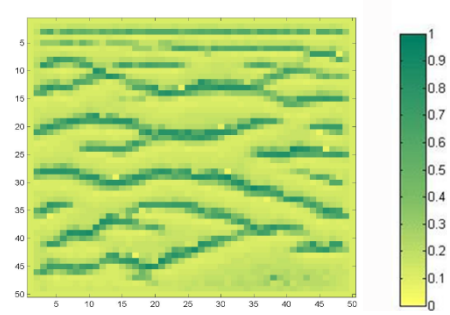

Fig. 20. Effect of soil moisture redistribution due to roots on pattern formation. (A) No roots redistribution. (B) Roots are able to reroute the soil moisture from adjacent less vegetated areas.

\subsubsection{Impact of local interactions}

The effect of interactions between adjacent clumps of vegetation was modeled, as indicated before, by allowing the transpiration efficiency of the vegetation at a certain pixel to depend on the vegetal density of the nearby pixels (see Eq. 7). We explored the effect of this spatial interaction on pattern formation by examining the evolution of the system in its absence, and comparing it with the results obtained for the base conditions, which includes it. Figure 19 shows that even in the absence of spatial interactions between plants in adjacent pixels, the system evolves towards a patterned configuration. However, the shape of the pattern and the total amount of vegetation arising in the domain are different in the two cases, as also evident in Fig. 22. This suggests that the net effect of the spatial interactions encoded in Eq. (8) through Eq. (12) (protection from temperature and humidity fluctuations, protection from soil erosion, protection from the mechanical damages due to winds and animals, enhancement of soil fertility through litter formation and nutrient replenishment and so on) is important for the ultimate configuration of patterns and total amount of biomass produced, although not essential for the emergence of the patterns themselves.

In order to investigate the individual effect of each of the interactions of Eq. (7), we carried out five simulations of the system, each one performed by setting one of the coefficients $\alpha_{i}$ of Eq. (8) through Eq. (12) equal to zero. A statistical analysis of the vegetation clusters obtained in those five cases is compared to the base conditions in Table 2. Notably, the simulation obtained with $\alpha_{2}=0$, which corresponds to the absence of facilitation due to surrounding vegetation, does not lead to the formation of patterns, whereas all the other cases do; the emerging banded patterns are of different shape and dimension, although never in a measure that results in the transition to spots or labyrinths, at least for the set of soil-climatic conditions considered here.

\subsubsection{Impact of soil moisture redistribution due to roots}

In order to investigate the role of the soil moisture redistribution due to the presence of roots, we compare the spatial 
A)

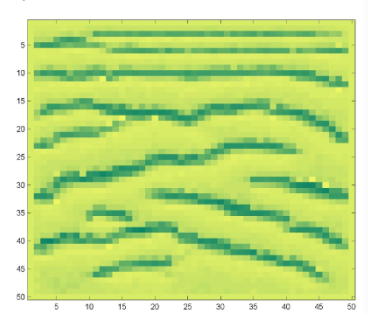

B)

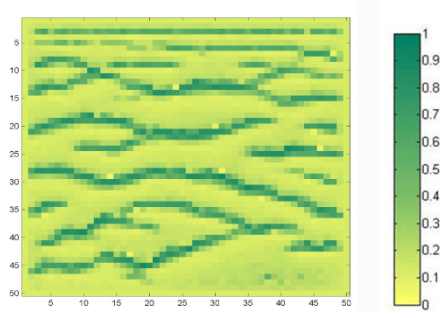

Fig. 21. Effect of spatially variable reflectance on pattern formation. (A) Uniform reflectance, that is, constant albedo. (B) Spatially variable albedo.

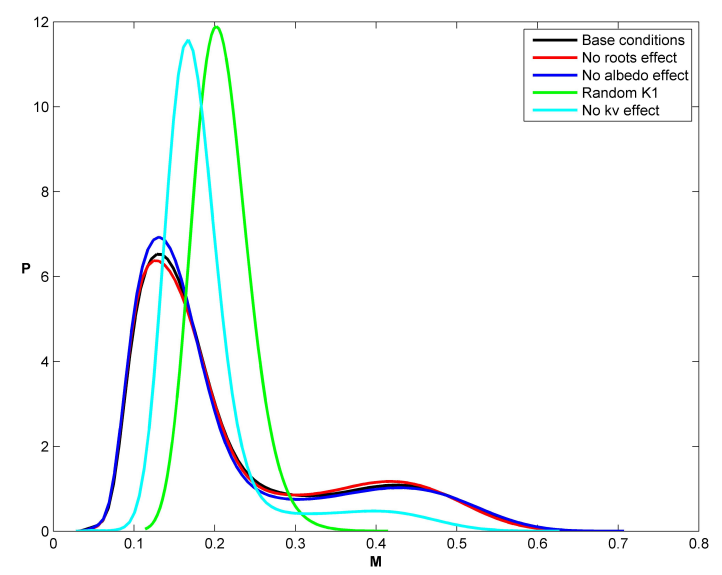

Fig. 22. PDFs of the vegetation density for the cases of: base conditions (black); no water redistribution due to roots (red); no spatially variable albedo (blue); hydraulic conductivity randomly distributed in space and independent from the vegetal density (green); no lateral facilitation and competition effects through transpiration efficiency (cyan).

distribution of vegetation obtained by allowing vegetation from each pixel to extract water from up to $12.5 \%$ of the area of each of the four adjacent pixels $\left(\xi_{R}=1.5\right)$ with the one resulting from neglecting this effect $\left(\xi_{R}=0\right)$. Results of this comparison are presented in Fig. 20. It is noticeable that the patterns that arise when this effect is neglected are nearly indistinguishable from the ones arising in the base conditions case. However, a deeper analysis (Fig. 22) shows that the PDF of the vegetation density obtained in the case where, due to roots, no competition for available soil moisture is considered, has a lower mode for the bare soil interval than the base condition case, with an average vegetation (average of the pixel vegetation density across the domain) being roughly equal $(0.209$ against 0.208$)$. Since the magnitude of this effect is much lower than the cases previously analyzed, the overall conclusion is that roots effect has a minor promoting effect on the formation of patterns.

\subsubsection{Impact of albedo}

Modeling the soil reflectance as a function of soil moisture content was aimed at incorporating the mutual effect that spatially variable water fluxes and vegetation have on the local potential rate of evapotranspiration. Figure 21 presents a comparison between a case in which this effect is modeled as proposed in Eq. (4); the case in which this effect is neglected, the reflectance being set constant in space and equal to the average of the values of reflectance of each pixel of the base condition case. Although a spatially variable reflectance determines a spatially variable absorbed net radiation and, thus, affects the energy budget of the entire hillslope, the patterns obtained in the two cases described above are qualitatively indistinguishable. The analysis of the PDF of vegetal density shown in Fig. 22, however, suggests that the vegetal configuration over the hillslope deviates from the base condition. In particular, the fact that the PDF obtained with a constant albedo shows higher modes (for both low vegetation and high vegetation) than the base condition case, indicates that a net reflectance that increases as soil moisture decreases has a minor negative impact on the formation of patterns. Given that both distributions are bimodal and that that patterns are clearly present in both, the case with higher modes corresponds to the one where the patterns are more marked, since it is more likely for the vegetation density to take values close to the two modes (representing the bare soil conditions and the vegetated patches).

\section{Summary and conclusions}

In this study we proposed a mechanistic modeling of those hydro-thermo-dynamics that, at the hillslope scale, are responsible for the phenomenon of vegetation pattern formation and evolution. The model performs the water and energy balance of a hillslope and accounts for vertical and horizontal water fluxes. The dynamics, inducing the emergence of vegetation patterns, are explicitly identified and modeled and their individual impact on the phenomenon is quantified.

Our results show that the proposed model is able to reproduce the types of naturally occurring patterns referred to as bands, spots and labyrinths. The model was satisfactorily validated by comparing simulations with observed natural patterns in the areas of Niger near Niamey and Somalia near Garoowe.

The analyses of the processes involved in the formation of patterns suggest that the phenomenon is primarily driven by run-on infiltration and local mechanisms of facilitation/competition existing among adjacent vegetation groups. Nonetheless, even in the presence of those mechanisms, patterns arise only when the climatic conditions, particularly annual precipitation and net radiation, are favorable. In particular, we found that, with decreasing precipitation or, conversely, increasing net radiation, the system drifts from 
fully vegetated with undistinguishable vegetation structures to self-organized patterns. When the behavior of the system is analyzed as a function of the potential humidity, which incorporates both the effect of precipitation and radiation, we found that patterns emerge when the potential humidity index is within a certain range (that for our simulated condition was found to be between 0.2 and 0.3 ), while no distinguishable patterns arise for climatic conditions too arid or too humid.

In the range of climatic conditions favorable for the formation of self-organized vegetation structures, the peculiar spatial features of patterns are determined by the characteristics of the spatial interactions induced by run-on infiltration, facilitation/inhibition dynamics between adjacent vegetation groups, effects of nutrient and litter transport and deposition, competition for soil moisture through roots, and effects of spatially inhomogeneous surface reflectance. Nonetheless, our study indicates that the surface run-on infiltration is the dominant dynamic. No self-organized structures, in fact, were observed in the absence of any surface runoff production and subsequent run-on infiltration; moreover, the system was found to be extremely sensitive to the relationship between vegetation density and soil permeability, that is, to the feedback between plants and soil. Only when that feedback is appropriate for the given set of forcing dynamics, can it trigger the process of surface runoff formation and run-on infiltration that is necessary to moisten some soil patches enough to promote further establishment of vegetation which, in turn, will further facilitate water infiltration. Although this is not the only process which could potentially lead to soil moisture redistribution in space (e.g., effect of roots or the effect of gradients in the hydraulic conductivity which is not considered in this study), it is no surprise that it is the one with the largest impact. In fact, both the simulations and the cited literature, indicate that the water input at each point in space can be several times larger than the mere precipitated water when the input from upslope is considered in the water budget. In a water-limited environment, this extra water input can make a great difference for vegetation establishment, provided that it can actually infiltrate the soil and not simply run downhill.

As for the impact of the other dynamics, we found that the effect of facilitation/competition due to vegetation interactions, soil erosion and nutrient transport has the highest impact on the phenomenon and has an influence on the ultimate shape of patterns; among those dynamics, the effect of facilitation due to the presence of surrounding vegetation was found to be the more significant. Effects of roots and albedo are comparatively less important but still have an impact on pattern definition, evolution and on the total vegetation coverage that establishes on the domain.

\section{Appendix}

The original water balance model of Eagleson is a onedimensional representation of soil moisture dynamics as forced by a stochastic climate (Eagleson, 1978e, d, c, b, a, $\mathrm{g}, \mathrm{f})$. The model describes the relationships between annual amounts of precipitation, surface and groundwater runoff, infiltration and evapotranspiration as a function of soil moisture and soil and vegetation characteristics. The description is physically based and only accounts for processes operating in the vertical direction across the soil-atmosphere interface. $\int_{0}^{t}\left\{i(t)-e_{\mathrm{T}}(t)-\frac{\partial}{\partial t}\left[V_{\mathrm{ss}}(t)-V_{\mathrm{rg}}(t)\right]\right\} \mathrm{d} t=\int_{0}^{t}\left[r_{\mathrm{s}}(t)+r_{\mathrm{g}}(t)\right] \mathrm{d} t$

The water balance equation for a control volume comprising a soil column and its surface is:

$$
\begin{gathered}
\int_{0}^{t}\left\{i(t)-e_{\mathrm{T}}(t)-\frac{\partial}{\partial t}\left[V_{\mathrm{ss}}(t)-V_{\mathrm{rg}}(t)\right]\right\} \cdot \mathrm{d} t \\
=\int_{0}^{t}\left[r_{\mathrm{s}}(t)+r_{\mathrm{g}}(t)\right] \cdot \mathrm{d} t,
\end{gathered}
$$

where $i(t), e_{\mathrm{T}}(t), V_{\mathrm{ss}}(t), V_{\mathrm{rg}}(t), r_{\mathrm{S}}(t)$ and $r_{\mathrm{g}}(t)$ are the instantaneous values of storm intensity, evapotranspiration rate, volume of water storage in the surface, volume of water storage in the subsurface, surface runoff rate, and groundwater runoff rate.

In order to obtain an analytical solution of Eq. (A.1), it is assumed that the system is in equilibrium with climate in its mean value. Therefore, taking the expectation of Eq. (A.1) leads to (Eagleson, 1978f):

$m_{\mathrm{PA}}-E\left[E_{\mathrm{TA}}\right]=E\left[R_{\mathrm{SA}}\right]+E\left[R_{\mathrm{gA}}\right]$

or, equivalently

$E\left[I_{\mathrm{A}}\right]=m_{\mathrm{PA}}-E\left[R_{\mathrm{SA}}\right]=E\left[E_{\mathrm{TA}}\right]+E\left[R_{\mathrm{A}}\right]$,

where $m_{\mathrm{PA}}, E\left[I_{\mathrm{A}}\right], E\left[E_{\mathrm{TA}}\right], E\left[R_{\mathrm{SA}}\right]$ and $E\left[R_{\mathrm{gA}}\right]$ are the expected volumes of precipitation, infiltration, actual evapotranspiration, surface runoff and groundwater runoff, respectively. Expressions for these water balance components (Eagleson, 1978b, c, d, e) are analytical functions of soil moisture and a small number of climatic, vegetation, and soil parameters, as listed in Table 1. Compact functional expressions of the main components of the water balance Eq. (A.2) as derived by Eagleson are repeated below. However, we refer the reader to the original papers (Eagleson, 1978a, b, c, d, $\mathrm{e}, \mathrm{f}, \mathrm{g}$ ) for detailed expressions.

Surface runoff:

$$
E\left[R_{\mathrm{SA}}\right]=\max \left\{0, m_{\mathrm{PA}} e^{-G-2 \sigma} \Gamma(\sigma+1) \sigma^{-\sigma}-E\left[E_{\mathrm{r}_{\mathrm{A}}}\right]\right\}
$$

Groundwater runoff:

$E\left[R_{\mathrm{g}_{\mathrm{A}}}\right]=m_{\tau} K(1) s_{\mathrm{o}}^{\mathrm{c}}-T w$ 
Capillary rise from the water table:

$w=K(1)\left[1+\frac{3 / 2}{\mathrm{mc}-1}\right]\left[\frac{\psi(1)}{Z}\right]^{\mathrm{mc}}$

Infiltration:

$$
E\left[I_{A}\right]=\left\{\begin{array}{c}
m_{\mathrm{PA}}\left[1-e^{-G-2 \sigma} \Gamma(\sigma+1) \sigma^{-\sigma}\right] \text { if } R_{\mathrm{S}_{\mathrm{A}}}>0 \\
m_{\mathrm{PA}} \text { otherwise }
\end{array},\right.
$$

where $\Gamma()$ is the Gamma function, and $G$ and $\sigma$ are the gravitational and the capillary infiltration parameters, respectively (Eagleson, 1978c, e).

Potential evapotranspiration:

$E\left[E_{\mathrm{PA}}\right]=m_{v} m_{\mathrm{tb}}\left[1-M\left(1-k_{v}\right)\right] e_{\mathrm{p}}$

Actual evapotranspiration:

$E\left[E_{\mathrm{T}_{\mathrm{A}}}\right]=\left\{\begin{array}{c}E\left[E_{\mathrm{PA}}^{*}\right] J\left(E_{\mathrm{e}}, M, k_{v}, 0\right) \text { if } R_{\mathrm{S}_{\mathrm{A}}}>0 \\ E\left[E_{\mathrm{PA}}\right] J\left(E_{\mathrm{e}}, M, k_{v}, h_{0}\right) \text { otherwise, }\end{array}\right.$

where $E\left[E_{\mathrm{PA}}^{*}\right]=E\left[E_{\mathrm{PA}}-E_{r_{\mathrm{A}}}\right]$ is potential evapotranspiration from soil moisture, and $E\left[E_{\mathrm{rA}}\right]$ is potential evaporation from surface retention.

The function $J()$ in Eq. (A.80) is the evapotranspiration efficiency function, that is, the ratio of actual to potential evapotranspiration. It depends on the bare soil evaporation effectiveness, $E_{\mathrm{e}}$, - itself a function of soil moisture, soil hydraulic characteristics, and vegetation characteristics (Eagleson, 1978d),

$J\left(E_{\mathrm{e}}, M, k_{v}, h_{\mathrm{o}}\right)=\frac{(1-M) E\left[E_{\mathrm{s}}\right]+M E\left[E_{v}\right]}{m_{\mathrm{tb}} e_{\mathrm{p}}\left(1-M+M k_{v}\right)}(A .9)$,

where $E\left[E_{\mathrm{s}}\right]$ is the actual evaporation from the bare soil fraction and $E\left[E_{v}\right]$ is actual transpiration from the vegetated fraction.

For a given climate, vegetation type and soil characteristics, the water balance Eq. (A.2) are functions of the average soil moisture content, $s_{\mathrm{o}}$, and the fractional vegetation coverage, $M$. For each soil, vegetation type and climate, the unique set of $s_{\mathrm{o}}$ and $M$ that satisfies (A.2) and closes the water balance is obtained by imposing the condition that the fractional vegetation coverage adjust to the current climate so as to minimize vegetation water stress while maximizing biomass production (Eagleson, 1978f).

Equation (A.2) through Eq. (A.9) characterize the mean water balance for a given soil, vegetation type and climate. These equations may also be used to characterize the water balance of any given year in a first order approximation by substituting annual values of precipitation and potential evapotranspiration for their corresponding means (Eagleson, 1978a, g).

Edited by: E. Zehe

\section{References}

Barbier, N., Couteron, P., Lefever, R., Deblauwe, V., and Lejeune, O.: Spatial decoupling of facilitation and competition at the origin of gapped vegetation patterns, Ecology, 89, 1521-1531, 2008.

Boaler, S. B. and Hodge, C. A. H.: Vegetation stripes in Somaliland, J. Ecol., 50, 465-524, 1962.

Borgogno, F., D’Odorico, P., Laio, F., and Ridolfi, L.: Mathematical models of vegetation pattern formation in ecohydrology, Rev. Geophys., 47, RG1005, doi:10.1029/2007RG000256, 2009.

Bromley, J., Brouwer, J., Barker, T., Gaze, S., and Valentin, C.: The role of surface water redistribution in an area of patterned vegetation in south west Niger, J. Hydrol., 198, 1-29, 1997.

Casenave, A. and Valentin, C.: A runoff capability classification system based on surface features criteria in semi-arid areas of West Africa, J. Hydrol., 130, 231-249, 1992.

D'Odorico, P., Laio. F., and Ridolfi, L.: Patterns as indicators of productivity enhancement by facilitation and competition in dryland vegetation, J. Geophys. Res.-Biogeosci., 111, G03010, doi:10.1029/2006JG000176, 2006.

Driessen, P., Deckers, J., Spaargaren, O., and Nachtergaele, F.: Lecture notes on the major soils of the world, World Soil Resources Reports 2000, 94, . vi + 334, ISSN 0532-0488, ISBN 92-5104637-9, Record Number 20023015720, 2001.

Dunkerley, D. and Brown, K.: Banded vegetation near Broken Hill, Australia: significance of surface roughness and soil physical properties, Catena, 37, 75-88, 1999.

Eagleson, P. S.: Climate, soil and vegetation.1. Introduction to water balance dynamics, Water Resour. Res., 14, 705-712, 1978a.

Eagleson, P. S.: Climate, soil and vegetation .2. Distribution of annual precipitation derived from observed storm sequences, Water Resour. Res., 14, 713-721, 1978b.

Eagleson, P. S.: Climate, soil and vegetation .3. Simplified model of soil moisture movement in liquid phase, Water Resour. Res., 14, 722-730, 1978c.

Eagleson, P. S.: Climate, soil and vegetation .4. Expected value of annual evapotranspiration, Water Resour. Res., 14, 731-739, 1978d.

Eagleson, P. S.: Climate, soil and vegetation .5. Derived distribution of storm surface runoff, Water Resour. Res., 14, 741-748, 1978e.

Eagleson, P. S.: Climate, soil and vegetation .6. Dynamics of annual waer balance, Water Resour. Res., 14, 749-764, $1978 f$.

Eagleson, P. S.: Climate, soil and vegetation .7. Derived distribution of annual water yield, Water Resour. Res., 14, 765-776, 1978g.

Greig-Smith, P.: Pattern in Vegetation, J. Ecol., 67, 755-779, 1979.

HilleRisLambers, R., Rietkerk, M., van den Bosh, F., Prins, H. H. T., and de Kroon, H.: Vegetation patter formation in semi-arid grazing systems, Ecology, 82, 50-61, 2001.

Holmgren, M., Sheffer, M., and Huston, M. A.: The interplay of facilitation and competition in plant communities, Ecology, 78, 1966-1975, 1997.

Jeltsch, F., Milton, S. J., Dean, W. R. J., and van Rooyen, N.: Tree spacing and coexistence in semiarid savannas, J. Ecol., 84, 583595, 1996.

Klausmeier, C. A.: Regular and irregular patterns in semi-arid vegetation, Science, 284, 1826-1828, 1999.

Lefever, R. and Lejeune, O.: On the origin of tiger bush, B. Math. Biol., 59, 263-294, 1997. 
Leprun, J.-C.: The influences of ecological factors on tiger bush and dotted bush patterns along a gradient from Mali to northern Burkina Faso, Catena, 37 25-44, 1999.

Lobell, D. B. and Asner, G. P.: Moisture Effects on Soil Reflectance, Soil Sci. Soc. Am. J., 66, 722-727, 2002.

Muchiri, P. W.: Climate of Somalia, FAO-SWALIM, Nairobi, 2007.

Rechid, D., Raddatz, T. J., and Jacob, D.: Parameterization of snowfree land surface albedo as a function of vegetation phenology based on MODIS data and applied in climate modelling, Theor. Appl. Climatol., 95, 245-255, 2009.

Rietkerk, M., Boerlijst, M. C., Van Langevelde, F., HilleRisLambers, R., Van de Koppel, J., Kumar, L., Prins, H. H. T., and De Roos, A. M.: Self-organization of vegetation in arid ecosystemns, The American Naturalist, 160, 524-530, 2002.

Rietkerk, M. and Van de Koppel, J.: Regular pattern formation in real ecosystems, Trends. Ecol. Evol., 23, 169-175, doi:10.1016/j.tree.2007.10.013, 2008

Saco, P. M., Willgoose, G. R., and Hancock, G. R.: Ecogeomorphology of banded vegetation patterns in arid and semi-arid regions, Hydrol. Earth Syst. Sci., 11, 1717-1730, doi:10.5194/hess-11-1717-2007, 2007.

Schymanski, S. J., Silvapalan, M., Roderick, M. L., Hutley, L. B., and Beringer, J.: An optimalitybased model of the dynamic feedbacks between natural vegetation and the water balance, 25, Water Resour. Res., 45, W01412, doi:10.1029/2008WR006841, 2009.

Schymanski, S. J., Kleidon, A., Stieglitz, M., and Narula, J.: Maximum entropy production allows a simple representation of heterogeneity in semiarid ecosystems, Trans. R. Soc. Lond. B. Biol. Sci., 365, 1449-1455, doi:10.1098/rstb.2009.0309, 2010.
Sherrat, J. A.: An analysis of vegetation stripe formation in semiarid landscapes, J. Math. Biol., 51, 183-197, 2005.

Thiéry, J., d'Herbès, J.-M., and Valentin, C.: A model for simulating the genesis of banded patterns in Niger, J. Ecol., 83, 497-507, 1995.

Tietjen, B., Jeltsch, F., Zehe, E., Classen, N., Groengroeft, A., Schiffers, K., and Oldeland, J.: Effects of climate change on the coupled dynamics of water and vegetation in drylands, Ecohydrol., 3, 226-237, doi:10.1002/eco.70, 2010.

Valentin, C., d'Herb'es, J. M., and Poesen, J.: Soil and water components of banded vegetation patterns, Catena, 37, 1-24, doi:10.1016/S0341-8162(99)00053-3, 1999.

Venema, J. H.: Land resources assessment of Somalia, FAOSWALIM, Nairobi, 2007.

Wang, K., Wang, P., Liu, J., Sparrow, M., Haginoya, S., and Zhou, X.: Variation of surface albedo and soil thermal paramenters with soil moisture content at a semi-desert site on the western Tibetan plateau, Bound.-Layer Meteorol., 116, 117-129, 2005.

Wiegand, K., Jeltsch, F., and Ward, D.: Analysis of the population dynamics of Acacia trees in the Negev desert, Israel with a spatially-explicit computer simulation model, Ecol. Modell., 117, 203-224, 1999.

Wiegand, K., Jeltsch, F., and Ward, D.: Do spatial effects play a role in the spatial distribution of desert-dwelling Acacia raddiana, $\mathbf{J}$ Veget. Sci., 11, 473-484, 2000.

Worral, G. A.: The Butana grass patterns, J. Soil Sci. 10, 34-53, 1959.

Worral, G. A.: Patchiness in vegetation in the northern Sudan, J. Ecol., 48, 107-117, 1960. 